\title{
TENSOR PRODUCT STRUCTURE OF AFFINE DEMAZURE MODULES AND LIMIT CONSTRUCTIONS
}

\author{
G. FOURIER* AND P. LITTELMANN*
}

\begin{abstract}
Let $\mathfrak{g}$ be a simple complex Lie algebra, we denote by $\widehat{\mathfrak{g}}$ the affine Kac-Moody algebra associated to the extended Dynkin diagram of $\mathfrak{g}$. Let $\Lambda_{0}$ be the fundamental weight of $\widehat{\mathfrak{g}}$ corresponding to the additional node of the extended Dynkin diagram. For a dominant integral $\mathfrak{g}$-coweight $\lambda^{\vee}$, the Demazure submodule $V_{-\lambda \vee}\left(m \Lambda_{0}\right)$ is a $\mathfrak{g}$-module. We provide a description of the $\mathfrak{g}$-module structure as a tensor product of "smaller" Demazure modules. More precisely, for any partition of $\lambda^{\vee}=\sum_{j} \lambda_{j}^{\vee}$ as a sum of dominant integral $\mathfrak{g}$-coweights, the Demazure module is (as $\mathfrak{g}$-module) isomorphic to $\bigotimes_{j} V_{-\lambda_{j}^{\vee}}\left(m \Lambda_{0}\right)$. For the "smallest" case, $\lambda^{\vee}=\omega^{\vee}$ a fundamental coweight, we provide for $\mathfrak{g}$ of classical type a decomposition of $V_{-\omega} \vee\left(m \Lambda_{0}\right)$ into irreducible $\mathfrak{g}$-modules, so this can be viewed as a natural generalization of the decomposition formulas in [13] and [16]. A comparison with the $U_{q}(\mathfrak{g})$-characters of certain finite dimensional $U_{q}^{\prime}(\widehat{\mathfrak{g}})$-modules (Kirillov-Reshetikhin-modules) suggests furthermore that all quantized Demazure modules $V_{-\lambda \vee, q}\left(m \Lambda_{0}\right)$ can be naturally endowed with the structure of a $U_{q}^{\prime}(\widehat{\mathfrak{g}})$-module. We prove, in the classical case (and for a lot of non-classical cases), a conjecture by Kashiwara [10], that the "smallest" Demazure modules are, when viewed as $\mathfrak{g}$-modules, isomorphic to some KR-modules. For an integral dominant $\widehat{\mathfrak{g}}$-weight $\Lambda$ let $V(\Lambda)$ be the corresponding irreducible $\widehat{\mathfrak{g}}$-representation. Using the tensor product decomposition for Demazure modules, we give a description of the $\mathfrak{g}$-module structure of $V(\Lambda)$ as a semi-infinite tensor product of finite dimensional $\mathfrak{g}$-modules. The case of twisted affine Kac-Moody algebras can be treated in the same way, some details are worked out in the last section.
\end{abstract}

\section{Introduction}

Let $\mathfrak{g}$ be a simple complex Lie algebra, we denote by $\widehat{\mathfrak{g}}$ the affine KacMoody algebra associated to the extended Dynkin diagram of $\mathfrak{g}$. (The

Received July 5, 2005.

Revised November 21, 2005.

2000 Mathematics Subject Classification: 22E46, 14M15.

*This research has been partially supported by the EC TMR network "LieGrits", contract MRTN-CT 2003-505078. 
twisted case is considered separately in the last section.) Let $\Lambda_{0}$ be the fundamental weight of $\widehat{\mathfrak{g}}$ corresponding to the additional node of the extended Dynkin diagram. The basic representation $V\left(\Lambda_{0}\right)$ is one of the most important representations of $\widehat{\mathfrak{g}}$ because its structure determines strongly the structure of all other highest weight representations $V(\Lambda), \Lambda$ an arbitrary dominant integral weight for $\widehat{\mathfrak{g}}$.

Let $P^{\vee}$ be the coweight lattice of $\mathfrak{g}$. An element $\lambda^{\vee}$ in the coroot lattice, can be viewed as an element of the affine Weyl group $W^{\text {aff }}$ (see Section 1), and one can associate to $\lambda^{\vee}$ the Demazure submodule $V_{\lambda} \vee(\Lambda)$ of $V(\Lambda)$ (see Section 2).

Actually, this construction generalizes to arbitrary $\lambda^{\vee} \in P^{\vee}$ in the following way: one can write $\lambda^{\vee}$ as $w \sigma \in \widetilde{W}^{\text {aff }}$ in the extended affine Weyl group, where $w \in W^{\text {aff }}$ and $\sigma$ corresponds to an automorphism of the Dynkin diagram of $\widehat{\mathfrak{g}}$. Denote by $V_{\lambda^{\vee}}(\Lambda)$ the Demazure submodule $V_{w}\left(\Lambda^{\prime}\right)$ of the highest weight module $V\left(\Lambda^{\prime}\right)$, where $\Lambda^{\prime}=\sigma(\Lambda)$.

If $\lambda^{\vee}$ is a dominant coweight, then the Demazure module $V_{-\lambda} \vee\left(m \Lambda_{0}\right)$ is in fact a $\mathfrak{g}$-module, and it is interesting to study its structure as $\mathfrak{g}$-module. So one would like to get a restriction formula expressing $V_{-\lambda \vee}\left(m \Lambda_{0}\right)$ as a direct sum of simple $\mathfrak{g}$-representations. We write $\bar{V}_{-\lambda^{\vee}}\left(m \Lambda_{0}\right)$ for the Demazure module viewed as a $\mathfrak{g}$-module.

A first reduction step is the following theorem describing the Demazure module as a tensor product. Such a decomposition formula for Demazure modules was first observed by Sanderson [20] in the affine rank two case, and was later studied in the case of classical groups in the framework of perfect crystals for example in [14], see [6] for a more complete account. We provide in this article a description of the Demazure module as a tensor product of modules of the same type, but for "smaller coweights". More precisely, let $\lambda^{\vee}$ be a dominant coweight and suppose we are given a decomposition

$$
\lambda^{\vee}=\lambda_{1}^{\vee}+\lambda_{2}^{\vee}+\cdots+\lambda_{r}^{\vee}
$$

of $\lambda^{\vee}$ as a sum of dominant coweights. The following theorem is a generalization of a result in [16], where the statement has been proved in the case $m=1$ and under the additional assumption that all the $\lambda_{i}^{\vee}$ are minuscule fundamental weights, and the decomposition formulas in [4], [5], [13] and [14], where in the framework of perfect crystals for classical groups many cases have been discussed. (The corresponding version for a twisted Kac-Moody algebra can be found in Section 4.) 
THEOREM 1. For all $m \geq 1$, we have an isomorphism of $\mathfrak{g}$-representations between the Demazure module $\bar{V}_{-\lambda^{\vee}}\left(m \Lambda_{0}\right)$ and the tensor product of Demazure modules:

$$
\bar{V}_{-\lambda \vee}\left(m \Lambda_{0}\right) \simeq \bar{V}_{-\lambda_{1}^{\vee}}\left(m \Lambda_{0}\right) \otimes \bar{V}_{-\lambda_{2}^{\vee}}\left(m \Lambda_{0}\right) \otimes \cdots \otimes \bar{V}_{-\lambda_{r}^{\vee}}\left(m \Lambda_{0}\right) .
$$

Of course, to analyse the structure of $\bar{V}_{-\lambda \vee}\left(m \Lambda_{0}\right)$ as a $\mathfrak{g}$-module, the simplest way is to take a decomposition of $\lambda^{\vee}$ as a sum of fundamental coweights $\lambda^{\vee}=\sum a_{i} \omega_{i}^{\vee}$. So by Theorem 1, it remains to describe the structure of the $\bar{V}_{-\omega_{i}^{\vee}}\left(m \Lambda_{0}\right)$ as a $\mathfrak{g}$-module. We give such a description below for all fundamental coweights for the classical groups. For the exceptional groups we give the decomposition in the cases interesting for the limit constructions considered later. The enumeration of the fundamental weights is as in [1], we write $\omega_{0}$ for the trivial weight. For more details on the notation see Section 2, we only recall here that we use the abbreviations $V_{-\omega} \vee\left(m \Lambda_{0}\right)$ and $V_{-\nu\left(\omega^{\vee}\right)}\left(m \Lambda_{0}\right)$ for the Demazure submodule associated to the translation $t_{-\nu\left(\omega^{\vee}\right)}$, viewed as an element in the extended affine Weyl group. We write $V^{*}$ for the contragradient dual of a representation $V$. Many special cases of the list below have been calculated before, for example by the Kyoto school ([10], [13], [14], [21]). (The corresponding version for a twisted Kac-Moody algebra can be found in Section 4.)

Theorem 2. Let $\omega^{\vee}$ be a fundamental coweight and let $V_{-\omega} \vee\left(m \Lambda_{0}\right)$ be the associated Demazure module. Viewed as a $\mathfrak{g}$-module, $\bar{V}_{-\omega \vee}\left(m \Lambda_{0}\right)$ decomposes into the direct sum of irreducible $\mathfrak{g}$-modules as follows:

- Type $\mathrm{A}_{n}: \bar{V}_{-\omega_{i}^{\vee}}\left(m \Lambda_{0}\right)=\bar{V}_{-\omega_{i}}\left(m \Lambda_{0}\right) \simeq V\left(m \omega_{i}\right)^{*}$ as $\mathfrak{s l}_{n}$-module for all $i=1, \ldots, n$.

- Type $\mathrm{B}_{n}$ : Set $\theta=0$ for $i$ even and $\theta=1$ for $i$ odd, then we have for $1 \leq i<n$ :

$$
\begin{aligned}
\bar{V}_{-\omega_{i}}\left(m \Lambda_{0}\right) & =\bar{V}_{-\omega_{i}}\left(m \Lambda_{0}\right) \\
& \simeq \bigoplus_{a_{i}+a_{i-2}+\cdots+a_{\theta}=m} V\left(a_{i} \omega_{i}+a_{i-2} \omega_{i-2}+\cdots+a_{\theta} \omega_{\theta}\right)
\end{aligned}
$$

and for $i=n$ :

$$
\begin{aligned}
\bar{V}_{-\omega_{n}}\left(m \Lambda_{0}\right) & =\bar{V}_{-2 \omega_{n}}\left(m \Lambda_{0}\right) \\
& \simeq \bigoplus_{a_{n}+a_{n-2}+\cdots+a_{\theta}=m} V\left(2 a_{n} \omega_{n}+a_{n-2} \omega_{n-2}+\cdots+a_{\theta} \omega_{\theta}\right)
\end{aligned}
$$

as $\mathfrak{s o}_{2 n+1}$-module. 
- Type $\mathrm{C}_{n}$ : for $j<n$ we have

$$
\bar{V}_{-\omega_{j}^{\vee}}\left(m \Lambda_{0}\right)=\bar{V}_{-2 \omega_{j}}\left(m \Lambda_{0}\right) \simeq \bigoplus_{a_{1}+\cdots+a_{j} \leq m} V\left(2 a_{1} \omega_{1}+\cdots+2 a_{j} \omega_{j}\right)
$$

and for $j=n: \bar{V}_{-\omega_{n}^{\vee}}\left(m \Lambda_{0}\right)=\bar{V}_{-\omega_{n}}\left(m \Lambda_{0}\right) \simeq V\left(m \omega_{n}\right)$ as $\mathfrak{s p}_{n}$-module.

- Type $\mathrm{D}_{n}$ : Set $\theta=0$ for $i$ even and $\theta=1$ for $i$ odd, then we have for $2 \leq i \leq n-2$ :

$$
\begin{aligned}
\bar{V}_{-\omega_{i}^{\vee}}\left(m \Lambda_{0}\right) & =\bar{V}_{-\omega_{i}}\left(m \Lambda_{0}\right) \\
& \simeq \bigoplus_{a_{i}+a_{i-2}+\cdots+a_{\theta}=m} V\left(a_{i} \omega_{i}+a_{i-2} \omega_{i-2}+\cdots+a_{\theta} \omega_{\theta}\right)
\end{aligned}
$$

and for $i=1, n-1, n: \bar{V}_{-\omega_{i}^{\vee}}\left(m \Lambda_{0}\right)=\bar{V}_{-\omega_{i}}\left(m \Lambda_{0}\right) \simeq V\left(m \omega_{i}\right)^{*}$ as $\mathfrak{s o}_{2 n}$-module.

- Type $\mathrm{E}_{6}$ :

$$
\begin{aligned}
& \bar{V}_{-\omega_{i}^{\vee}}\left(m \Lambda_{0}\right)=\bar{V}_{-\omega_{i}}\left(m \Lambda_{0}\right) \simeq V\left(m \omega_{i}\right)^{*} \text { for } i=1,6 \\
& \bar{V}_{-\omega_{2}^{\vee}}\left(m \Lambda_{0}\right)=\bar{V}_{-\omega_{2}}\left(m \Lambda_{0}\right) \simeq \bigoplus_{r=0}^{m} V\left(r \omega_{2}\right)
\end{aligned}
$$

as $\mathrm{E}_{6}$-module.

- Type $\mathrm{E}_{7}$ :

$$
\begin{aligned}
& \bar{V}_{-\omega_{7}^{\vee}}\left(m \Lambda_{0}\right)=\bar{V}_{-\omega_{7}}\left(m \Lambda_{0}\right) \simeq V\left(m \omega_{7}\right) \\
& \bar{V}_{-\omega_{1}^{\vee}}\left(m \Lambda_{0}\right)=\bar{V}_{-\omega_{1}}\left(m \Lambda_{0}\right) \simeq \bigoplus_{r=0}^{m} V\left(r \omega_{1}\right)
\end{aligned}
$$

as $\mathrm{E}_{7}$-module.

- Type $\mathrm{E}_{8}: \bar{V}_{-\omega_{8}^{\vee}}\left(m \Lambda_{0}\right)=\bar{V}_{-\omega_{8}}\left(m \Lambda_{0}\right) \simeq \bigoplus_{r=0}^{m} V\left(r \omega_{8}\right)$ as $\mathrm{E}_{8}$-module.

- Type $\mathrm{F}_{4}$ :

$$
\bar{V}_{-\omega_{1}^{\vee}}\left(m \Lambda_{0}\right)=\bar{V}_{-\omega_{1}}\left(m \Lambda_{0}\right) \simeq \bigoplus_{r=0}^{m} V\left(r \omega_{1}\right)
$$

and

$$
\bar{V}_{-\omega_{4}^{\vee}}\left(m \Lambda_{0}\right)=\bar{V}_{-2 \omega_{4}}\left(m \Lambda_{0}\right) \simeq \bigoplus_{r+s \leq m} V\left(r \omega_{1}+s 2 \omega_{4}\right)
$$

as $\mathrm{F}_{4}$-module. 
- Type $\mathrm{G}_{2}: \bar{V}_{-\omega_{2}^{\vee}}\left(m \Lambda_{0}\right)=\bar{V}_{-\omega_{2}}\left(m \Lambda_{0}\right) \simeq \bigoplus_{r=0}^{m} V\left(r \omega_{2}\right)$ as $\mathrm{G}_{2}$-module.

There is a very interesting conjectural connection with certain $U_{q}^{\prime}(\widehat{\mathfrak{g}})$ modules. Here $U_{q}^{\prime}(\widehat{\mathfrak{g}})$ denotes the quantized affine algebra without derivation.

Let $K R\left(m \omega_{i}\right)$ be the Kirillov-Reshetikhin-module for a multiple of a fundamental weight of $\mathfrak{g}$, for the precise definition see [4], it is irreducible as $U_{q}^{\prime}(\widehat{\mathfrak{g}})$ and the highest weight, when viewed as a $U_{q}(\mathfrak{g})$-module, is $m \omega_{i}$. In [9] Kashiwara introduced the notion of a good $U_{q}^{\prime}(\widehat{\mathfrak{g}})$-module, which, roughly speaking, is an irreducible finite dimensional $U_{q}^{\prime}(\widehat{\mathfrak{g}})$-module with a crystal basis and a global basis, and he proved that the tensor product of good modules is a good module. It is conjectured that the KR-modules are good. For all fundamental $\mathfrak{g}$-weights $\omega_{i}$ Kashiwara constructed this irreducible finite-dimensional integrable $U_{q}^{\prime}(\widehat{\mathfrak{g}})$-module $K R\left(\omega_{i}\right)$ and showed that it is good and even more that the crystal is isomorphic to a certain generalized Demazure crystal as a $\mathfrak{g}$-crystal.

Let $c_{k}^{\vee}=\frac{a_{k}}{a_{k}^{V}}$ (for the definition of the $a_{k}$ see Section 1.1) and $l \in \mathbb{N}$. Let $K R\left(l c_{k}^{\vee} \omega_{k}\right)$ be the Kirillov-Reshetikhin-module for $U_{q}^{\prime}(\widehat{\mathfrak{g}})$ associated to the weight $l c_{k}^{\vee} \omega_{k}$. It is more generally conjectured that the $K R\left(l c_{k}^{\vee} \omega_{k}\right)$ the crystal is isomorphic to the crystal of a Demazure module, after omitting the 0-arrows in both crystals [10]. Chari and the Kyoto school have calculated for classical Lie-algebras and some fundamental weights for nonclassical Lie-algebras the decomposition of the Kirillov-Reshetikhin module $K R\left(l c_{k}^{\vee} \omega_{k}\right)$ into irreducible $U_{q}(\mathfrak{g})$-modules [2]. By comparing the $U_{q}(\mathfrak{g})$ structure of the Kirillov-Reshetikhin module $K R\left(l c_{k}^{\vee} \omega_{k}\right)$ with the list in Theorem 2 we conclude:

Corollary 1. In all cases stated in Theorem 2, the Demazure module $\left(\bar{V}_{-\omega^{\vee}, q}\left(l \Lambda_{0}\right)\right)$ and the Kirillov-Reshetikhin module $K R\left(l c_{k}^{\vee} \omega_{k}^{*}\right)$ are, as $U_{q}(\mathfrak{g})$-modules, isomorphic.

In particular, if $K R\left(l c_{k}^{\vee} \omega_{k}^{*}\right)$ has a crystal basis, then the crystal is isomorphic to the crystal of $\left(V_{-\omega_{k}^{\vee}}\left(l \Lambda_{0}\right)\right)$ after omitting the arrows with label zero. By using the $U_{q}(\mathfrak{g})$-module isomorphism, we see that (in the cases above) the quantized Demazure modules $V_{-\omega_{k}^{\vee}, q}\left(l \Lambda_{0}\right)$ can be equipped with the structure of an irreducible $U_{q}^{\prime}(\widehat{\mathfrak{g}})$-module. In fact, using the Theorem 1, we see that for classical groups all quantized Demazure modules $V_{-\lambda^{\vee}, q}\left(l \Lambda_{0}\right), \lambda^{\vee}$ a dominant coweight, can be equipped with the structure 
of an $U_{q}^{\prime}(\widehat{\mathfrak{g}})$-module. Of course, in the exceptional case the same argument shows that when $\lambda^{\vee}$ can be written as linear combinations of the fundamental weights listed in Theorem 2 , then again $V_{-\lambda^{\vee}, q}\left(l \Lambda_{0}\right)$ can be equipped with the structure of an $U_{q}^{\prime}(\widehat{\mathfrak{g}})$-module. This leads to the following:

Conjecture 1. Let $\mathfrak{g}$ be a semisimple Lie algebra, let $U_{q}(\widehat{\mathfrak{g}})$ be the associated untwisted quantum affine algebra and let $U_{q}^{\prime}(\widehat{\mathfrak{g}})$ be its subalgebra without derivation. For all dominant coweights $\lambda^{\vee}$ and for all $l>0$, the Demazure module $V_{-\lambda \vee}, q\left(l \Lambda_{0}\right)$ can be endowed with the structure of a $U_{q}^{\prime}(\widehat{\mathfrak{g}})$ module admitting a crystal basis. Its crystal graph is isomorphic to the crystal of the Demazure module, after omitting the arrows labelled with zero.

The tensor decomposition structure in Theorem 1 holds in the following more general situation. Let $\Lambda_{i}, 1 \leq i \leq n$, be a fundamental weight of $\widehat{\mathfrak{g}}$ such that the corresponding coweight $\omega_{i}^{\vee}$ is minuscule. Let $\lambda^{\vee}$ be a dominant coweight and suppose we are given a decomposition

$$
\lambda^{\vee}=\omega_{i}^{\vee}+\lambda_{2}^{\vee}+\cdots+\lambda_{r}^{\vee}
$$

of $\lambda^{\vee}$ as a sum of dominant coweights and denote $\omega_{i}^{*}$ the highest weight of the irreducible $\mathfrak{g}$-module $V\left(\omega_{i}\right)^{*}$.

TheOREM $1 \mathrm{~A}$. For all $m \geq 0$ and $s \geq 1$, we have an isomorphism of $\mathfrak{g}$-representations between the Demazure module $\bar{V}_{-\lambda^{\vee}}\left(m \Lambda_{0}+s \Lambda_{i}\right)$ and the tensor product of Demazure modules:

$$
\begin{aligned}
& \bar{V}_{-\lambda \vee}\left(m \Lambda_{0}+s \Lambda_{i}\right) \\
& \quad \simeq V\left(s \omega_{i}^{*}\right) \otimes \bar{V}_{-\lambda_{2}^{\vee}}\left((m+s) \Lambda_{0}\right) \otimes \cdots \otimes \bar{V}_{-\lambda_{r}}\left((m+s) \Lambda_{0}\right) .
\end{aligned}
$$

Let $\Lambda$ be an arbitrary dominant integral weight for $\widehat{\mathfrak{g}}$. The $\widehat{\mathfrak{g}}$-module $V(\Lambda)$ is the direct limit of the Demazure-modules $V_{-N \lambda} \vee(\Lambda)$ for some dominant, integral, nonzero coweight of $\mathfrak{g}$. We give a construction of the $\mathfrak{g}$ module $\bar{V}(\Lambda)$ as a direct limit of tensor products of Demazure modules. This has been done before in the case of classical Lie-algebras for $\Lambda=r \Lambda_{0}$ (and corresponding weights obtained by automorphisms as in the statement of Theorem 2) by Kang, Kashiwara, Kuniba, Misra et al. [6], [13] via the theory of perfect crystals. In addition they have also considered some special weights in the case of non-classical groups. For $\mathrm{G}_{2}$, such a construction 
has been given by Yamane [21]. For the Lie algebras of type $E_{6}$ and $E_{7}$ a construction (only for the case $\Lambda=\Lambda_{0}$ ) was given by Peter Magyar [16] using the path model.

We provide in this article such a direct limit construction for arbitrary simple Lie algebras $\mathfrak{g}$. Let $\Lambda$ be a dominant, integral weight for $\widehat{\mathfrak{g}}$, then we can write $\Lambda=r \Lambda_{0}+\lambda$ with $\lambda$ dominant, integral for $\mathfrak{g}$.

Let $W$ be the $\mathfrak{g}$-module $W:=\bar{V}_{-\theta^{\vee}}\left(r \Lambda_{0}\right)$, where $\theta$ is the highest root of $\mathfrak{g}$, we show that $W$ contains a unique one-dimensional submodule. Fix $w \neq 0$ a $\mathfrak{g}$-invariant vector in $W$. Let $V(\lambda)$ be the irreducible $\mathfrak{g}$-module with highest weight $\lambda$ and define the $\mathfrak{g}$-module $V_{\lambda, r}^{\infty}$ to be the direct limit of:

$V_{\lambda, r}^{\infty}: V(\lambda) \hookrightarrow W \otimes V(\lambda) \hookrightarrow W \otimes W \otimes V(\lambda) \hookrightarrow W \otimes W \otimes W \otimes V(\lambda) \hookrightarrow \cdots$

where the inclusions are always given by taking a vector $u$ to its tensor product $u \mapsto w \otimes u$ with the fixed $\mathfrak{g}$-invariant vector in $W$.

Recall the notation $\bar{V}(\Lambda)$ for $V(\Lambda)$ viewed as a $\mathfrak{g}$-module. (The corresponding version for a twisted Kac-Moody algebra can be found in Section 4.)

THEOREM 3. For any integral dominant weight $\Lambda$ of $\widehat{\mathfrak{g}}, \Lambda=r \Lambda_{0}+\lambda$, the $\mathfrak{g}$-modules $V_{\lambda, r}^{\infty}$ and $\bar{V}(\Lambda)$ are isomorphic.

Remark 1. The choice of $W$ is convenient because it avoids case by case considerations. But, in fact, one could choose any other module $W=$ $V_{-\mu^{\vee}}\left(r \Lambda_{0}\right)^{\otimes m}$, where $V_{-\mu^{\vee}}\left(r \Lambda_{0}\right)$ is the Demazure module for a dominant, integral, nonzero coweight $\mu^{\vee}$ and $m$ is such that $V_{-\mu^{\vee}}\left(r \Lambda_{0}\right)^{\otimes m}$ contains a one-dimensional submodule.

\section{§1. The affine Kac-Moody algebra}

\subsection{Notations and basics}

In this section we fix the notation and the usual technical padding. Let $\mathfrak{g}$ be a simple complex Lie algebra. We fix a Cartan subalgebra $\mathfrak{h}$ in $\mathfrak{g}$ and a Borel subalgebra $\mathfrak{b} \supseteq \mathfrak{h}$. Denote $\Phi \subseteq \mathfrak{h}^{*}$ the root system of $\mathfrak{g}$, and, corresponding to the choice of $\mathfrak{b}$, let $\Phi^{+}$be the set of positive roots and let $\Delta=\left\{\alpha_{1}, \ldots, \alpha_{n}\right\}$ be the corresponding basis of $\Phi$.

For a root $\beta \in \Phi$ let $\beta^{\vee} \in \mathfrak{h}$ be its coroot. The basis of the dual root system (also called the coroot system) $\Phi^{\vee} \subset \mathfrak{h}$ is denoted $\Delta^{\vee}=\left\{\alpha_{1}^{\vee}, \ldots, \alpha_{n}^{\vee}\right\}$. 
We denote throughout the paper by $\Theta=\sum_{i=1}^{n} a_{i} \alpha_{i}$ the highest root of $\Phi$, by $\Theta^{\times}=\sum_{i=1}^{n} a_{i}^{\times} \alpha_{i}^{\vee}$ the highest root of $\Phi^{\vee}$ and by $\Theta^{\vee}=\sum_{i=1}^{n} a_{i}^{\vee} \alpha_{i}^{\vee}$ the coroot of $\Theta$. Note that $\Theta^{\vee} \neq \Theta^{\times}$in general. The Weyl group $W$ of $\Phi$ is generated by the simple reflections $s_{i}=s_{\alpha_{i}}$ associated to the simple roots.

Let $P$ be the weight lattice of $\Phi$ and let $P^{\vee}$ be the weight lattice of the dual root system $P^{\vee}$. Denote $P^{+} \subset P$ the subset of dominant weights and let $\mathbb{Z}[P]$ be the group algebra of $P$. For a simple root $\alpha_{i}$ let $\omega_{i}$ be the corresponding fundamental weight, we use the same notation for simple coroots and coweights. Recall that $\omega_{i}$ is called minuscule if $a_{i}^{\times}=1$, and the coweight $\omega_{i}^{\vee}$ is called minuscule if $a_{i}=1$.

Denote by $\mathfrak{h}_{\mathbb{R}} \subset \mathfrak{h}$ the real span of the coroots and let $\mathfrak{h}_{\mathbb{R}}^{*} \subset \mathfrak{h}^{*}$ be the real span of the fundamental weights. We fix a $W$-invariant scalar product $(\cdot, \cdot)$ on $\mathfrak{h}$ and normalize it such that the induced isomorphism $\nu: \mathfrak{h}_{\mathbb{R}} \rightarrow \mathfrak{h}_{\mathbb{R}}^{*}$ maps $\Theta^{\vee}$ to $\Theta$. With the notation as above it follows for the weight lattice $P^{\vee}$ of the dual root system $\Phi^{\vee}$ that

$$
\nu\left(\alpha_{i}^{\vee}\right)=\frac{a_{i}}{a_{i}^{\vee}} \alpha_{i} \quad \text { and } \quad \nu\left(\omega_{i}^{\vee}\right)=\frac{a_{i}}{a_{i}^{\vee}} \omega_{i}, \quad \forall i=1, \ldots, n \text {. }
$$

Let $\widehat{\mathfrak{g}}$ be the affine Kac-Moody algebra corresponding to the extended Dynkin diagram of $\mathfrak{g}$ (see $[7$, Chapter 7$]$ ):

$$
\widehat{\mathfrak{g}}=\mathfrak{g} \otimes_{\mathbb{C}} \mathbb{C}\left[t, t^{-1}\right] \oplus \mathbb{C} K \oplus \mathbb{C} d .
$$

Here $d$ denotes the derivation $d=t \frac{d}{d t}$ and $K$ is the canonical central element. The Lie algebra $\mathfrak{g}$ is naturally a subalgebra of $\widehat{\mathfrak{g}}$. In the same way, $\mathfrak{h}$ and $\mathfrak{b}$ are subalgebras of the Cartan subalgebra $\widehat{\mathfrak{h}}$ respectively the Borel subalgebra $\widehat{\mathfrak{b}}$ of $\widehat{\mathfrak{g}}$ :

$$
\widehat{\mathfrak{h}}=\mathfrak{h} \oplus \mathbb{C} K \oplus \mathbb{C} d, \quad \widehat{\mathfrak{b}}=\mathfrak{b} \oplus \mathbb{C} K \oplus \mathbb{C} d \oplus \mathfrak{g} \otimes_{\mathbb{C}} t \mathbb{C}[t] .
$$

Denote by $\widehat{\Phi}$ the root system of $\widehat{\mathfrak{g}}$ and let $\widehat{\Phi}^{+}$be the subset of positive roots. The positive non-divisible imaginary root in $\widehat{\Phi}^{+}$is denoted $\delta$. The simple roots are $\widehat{\Delta}=\left\{\alpha_{0}\right\} \cup \Delta$ where $\alpha_{0}=\delta-\Theta$. Let $\Lambda_{0}, \ldots, \Lambda_{n}$ be the corresponding fundamental weights, then for $i=1, \ldots, n$ we have

$$
\Lambda_{i}=\omega_{i}+a_{i}^{\vee} \Lambda_{0}
$$

The decomposition of $\widehat{\mathfrak{h}}$ in (1) has its corresponding version for the dual space $\widehat{\mathfrak{h}}^{*}$ :

$$
\widehat{\mathfrak{h}}^{*}=\mathfrak{h}^{*} \oplus \mathbb{C} \Lambda_{0} \oplus \mathbb{C} \delta,
$$


here the elements of $\mathfrak{h}^{*}$ are extended trivially, $\left\langle\Lambda_{0}, \mathfrak{h}\right\rangle=\left\langle\Lambda_{0}, d\right\rangle=0$ and $\left\langle\Lambda_{0}, K\right\rangle=1$, and $\langle\delta, \mathfrak{h}\rangle=\langle\delta, K\rangle=0$ and $\langle\delta, d\rangle=1$. Let $\widehat{\Delta}^{\vee}=\left\{\alpha_{0}^{\vee}, \alpha_{1}^{\vee}, \ldots\right.$, $\left.\alpha_{n}^{\vee}\right\} \subset \widehat{\mathfrak{h}}$ be the corresponding basis of the coroot system, then $\alpha_{0}^{\vee}=K-\Theta^{\vee}$.

Set $\widehat{\mathfrak{h}}_{\mathbb{R}}^{*}=\mathbb{R} \delta+\sum_{i=0}^{n} \mathbb{R} \Lambda_{i}$, by (3) and (2) we have $\mathfrak{h}_{\mathbb{R}}^{*} \subseteq \widehat{\mathfrak{h}}_{\mathbb{R}}^{*}$. The affine Weyl group $W^{\text {aff }}$ is generated by the reflections $s_{0}, s_{1}, \ldots, s_{n}$, where again $s_{i}=s_{\alpha_{i}}$ for a simple root. The cone $\widehat{C}=\left\{\Lambda \in \widehat{\mathfrak{h}}_{\mathbb{R}}^{*} \mid\left\langle\Lambda, \alpha_{i}^{\vee}\right\rangle \geq 0, i=\right.$ $0, \ldots, n\}$ is the fundamental Weyl chamber for $\widehat{\mathfrak{g}}$.

We put $\mathrm{a}^{\widehat{ }}$ on (almost) everything related to $\widehat{\mathfrak{g}}$. Let $\widehat{P}$ be the weight lattice of $\widehat{\mathfrak{g}}$, let $\widehat{P}^{+}$be the subset of dominant weights and let $\mathbb{Z}[\widehat{P}]$ be the group algebra of $\widehat{P}$. Recall the following properties of $\delta$ (see for example [7, Chapter 6]):

$$
\begin{aligned}
\left\langle\delta, \alpha_{i}^{\vee}\right\rangle= & 0 \forall i=0, \ldots, n, \quad w(\delta)=\delta \forall w \in W^{\mathrm{aff}}, \\
& \left\langle\alpha_{0}, \alpha_{i}^{\vee}\right\rangle=-\left\langle\Theta, \alpha_{i}^{\vee}\right\rangle \text { for } i \geq 1 .
\end{aligned}
$$

Put $a_{0}=a_{0}^{\vee}=1$ and let $A=\left(a_{i, j}\right)_{0 \leq i, j \leq n}$ be the (generalized) Cartan matrix of $\widehat{\mathfrak{g}}$. We have a non-degenerate symmetric bilinear form $(\cdot, \cdot)$ on $\widehat{\mathfrak{h}}$ defined by $([7$, Chapter 6$])$

$$
\begin{cases}\left(\alpha_{i}^{\vee}, \alpha_{j}^{\vee}\right)=\frac{a_{j}}{a_{j}^{\vee}} a_{i, j} & i, j=0, \ldots, n \\ \left(\alpha_{i}^{\vee}, d\right)=0 & i=1, \ldots, n \\ \left(\alpha_{0}^{\vee}, d\right)=1 & (d, d)=0 .\end{cases}
$$

The corresponding isomorphism $\nu: \widehat{\mathfrak{h}} \rightarrow \widehat{\mathfrak{h}}^{*}$ maps

$$
\nu\left(\alpha_{i}^{\vee}\right)=\frac{a_{i}}{a_{i}^{\vee}} \alpha_{i}, \quad \nu(K)=\delta, \quad \nu(d)=\Lambda_{0} .
$$

Denote by $\mathfrak{g}_{\mathrm{sc}}$ the subalgebra of $\widehat{\mathfrak{g}}$ generated by $\mathfrak{g}$ and $\alpha_{0}^{\vee}=K-\Theta^{\vee}$, then $\mathfrak{h}_{\mathrm{sc}}=\mathfrak{h} \oplus \mathbb{C} K$ is a Cartan subalgebra of $\mathfrak{g}_{\mathrm{sc}}$. The inclusion $\mathfrak{h}_{\mathrm{sc}} \rightarrow \widehat{\mathfrak{h}}$ induces an epimorphism $\widehat{\mathfrak{h}}^{*} \rightarrow \mathfrak{h}_{\mathrm{sc}}^{*}$ with one dimensional kernel. Now (4) implies that we have in fact an isomorphism

$$
\widehat{\mathfrak{h}}^{*} / \mathbb{C} \delta \longrightarrow \mathfrak{h}_{\mathrm{sc}}^{*}
$$

and we set $\mathfrak{h}_{\mathrm{sc}, \mathbb{R}}^{*}=\widehat{\mathfrak{h}}_{\mathbb{R}}^{*} / \mathbb{R} \delta$. Since $\mathbb{R} \delta \subset \widehat{C}$, we use the same notation $\widehat{C}$ for the image in $\mathfrak{h}_{\mathrm{sc}, \mathbb{R}}^{*}$. In the following we are mostly interested in characters of $\mathfrak{g}$-modules respectively $\mathfrak{g}_{\mathrm{sc}}$-modules obtained by restriction from $\widehat{\mathfrak{g}}$-modules, so we consider also the ring

$$
\mathbb{Z}\left[P_{\mathrm{sc}}\right]:=\mathbb{Z}[\widehat{P}] / I_{\delta},
$$

where $I_{\delta}=\left(1-e^{\delta}\right)$ is the ideal in $\mathbb{Z}[\widehat{P}]$ generated by $\left(1-e^{\delta}\right)$. 


\subsection{The extended affine Weyl group}

Since $W^{\text {aff }}$ fixes $\delta$, the group can be defined as the subgroup of $G L\left(\mathfrak{h}_{\mathrm{sc}, \mathbb{R}}^{*}\right)$ generated by the induced reflections $s_{0}, \ldots, s_{n}$.

Let $M \subset \mathfrak{h}_{\mathbb{R}}^{*}$ be the lattice $M=\nu\left(\bigoplus_{i=1}^{n} \mathbb{Z} \alpha_{i}^{\vee}\right)$. If $\mathfrak{g}$ is simply laced, then $M$ is the root lattice in $\mathfrak{h}_{\mathbb{R}}^{*}$, otherwise $M$ is the lattice in $\mathfrak{h}_{\mathbb{R}}^{*}$ generated by the long roots. An element $\Lambda \in \mathfrak{h}_{\mathrm{sc}, \mathbb{R}}^{*}$ can be uniquely decomposed into $\Lambda=\lambda+b \Lambda_{0}$ such that $\lambda \in \mathfrak{h}_{\mathbb{R}}^{*}$. For an element $\mu \in M$ let $t_{\mu} \in G L\left(\mathfrak{h}_{\mathrm{sc}, \mathbb{R}}^{*}\right)$ be the map defined by

$$
\Lambda=\lambda+b \Lambda_{0} \longmapsto t_{\mu}(\Lambda)=\lambda+b \Lambda_{0}+b \mu=\Lambda+\langle\Lambda, K\rangle \mu .
$$

Obviously we have $t_{\mu} \circ t_{\mu^{\prime}}=t_{\mu+\mu^{\prime}}$, denote $t_{M}$ the abelian subgroup of $G L\left(\mathfrak{h}_{\mathrm{sc}, \mathbb{R}}^{*}\right)$ consisting of the elements $t_{\mu}, \mu \in M$. Then $W^{\text {aff }}$ is the semidirect product $W^{\text {aff }}=W \ltimes t_{M}$.

The extended affine Weyl group $\widetilde{W}^{\text {aff }}$ is the semidirect product $\widetilde{W}^{\text {aff }}=$ $W \ltimes t_{L}$, where $L=\nu\left(\bigoplus_{i=1}^{n} \mathbb{Z} \omega_{i}^{\vee}\right)$ is the image of the coweight lattice. The action of an element $t_{\mu}, \mu \in L$, is defined as above in (6). $\widehat{C}$ :

Let $\Sigma$ be the subgroup of $\widetilde{W}^{\text {aff }}$ stabilizing the dominant Weyl chamber

$$
\Sigma=\left\{\sigma \in \widetilde{W}^{\text {aff }} \mid \sigma(\widehat{C})=\widehat{C}\right\} .
$$

Then $\Sigma$ provides a complete system of coset representatives of $\widetilde{W}$ aff $/ W^{\text {aff }}$ and $\widetilde{W^{a f f}}=\Sigma \ltimes W^{\text {aff. }}$. The elements $\sigma \in \Sigma$ are all of the form (one can verify this easily or see [1])

$$
\sigma=\tau_{i} t_{-\nu\left(\omega_{i}^{\vee}\right)}=\tau_{i} t_{-\omega_{i}},
$$

where $\omega_{i}^{\vee}$ is a minuscule coweight and $\tau_{i}=w_{0} w_{0, i}$, where $w_{0}$ is the longest word in $W$ and $w_{0, i}$ is the longest word in $W_{\omega_{i}}$, the stabilizer of $\omega_{i}$ in $W$.

We extend the length function $\ell: W^{\text {aff }} \rightarrow \mathbb{N}$ to a length function $\ell: \widetilde{W}^{\text {aff }} \rightarrow \mathbb{N}$ by setting $\ell(\sigma w)=\ell(w)$ for $w \in W^{\text {aff }}$ and $\sigma \in \Sigma$.

\section{§. Demazure modules}

\subsection{Definitions}

For a dominant weight $\Lambda \in \widehat{P}^{+}$let $V(\Lambda)$ be the (up to isomorphism) unique irreducible $\widehat{\mathfrak{g}}$-highest weight module of highest weight $\Lambda$.

Let $U(\widehat{\mathfrak{b}})$ be the enveloping algebra of the Borel subalgebra $\widehat{\mathfrak{b}} \subset \widehat{\mathfrak{g}}$. Given an element $w \in W^{\text {aff }} / W_{\Lambda}$, fix a generator $v_{w(\Lambda)}$ of the line $V(\Lambda)_{w(\Lambda)}=$ $\mathbb{C} v_{w(\Lambda)}$ of $\widehat{\mathfrak{h}}$-eigenvectors in $V(\Lambda)$ of weight $w(\Lambda)$. 
Definition 1. The $U(\widehat{\mathfrak{b}})$-submodule $V_{w}(\Lambda)=U(\widehat{\mathfrak{b}}) \cdot v_{w(\Lambda)}$ generated by $v_{w(\Lambda)}$ is called the Demazure submodule of $V(\Lambda)$ associated to $w$.

To associate more generally to every element $\sigma w \in \widetilde{W}^{\text {aff }}=\Sigma \ltimes W^{\text {aff }}$ a Demazure module, recall that elements in $\Sigma$ correspond to automorphisms of the Dynkin diagram of $\widehat{\mathfrak{g}}$, and thus define an associated automorphism of $\widehat{\mathfrak{g}}$, also denoted $\sigma$. For a module $V$ of $\widehat{\mathfrak{g}}$ let $V^{\sigma}$ be the module with the twisted action $g \circ v=\sigma^{-1}(g) v$. Then for the irreducible module of highest weight $\Lambda \in \widehat{P}^{+}$we get $V(\Lambda)^{\sigma}=V(\sigma(\Lambda))$.

So for $\sigma w \in \widetilde{W}^{\text {aff }}=\Sigma \ltimes W^{\text {aff }}$ we set

$$
V_{w \sigma}(\Lambda)=V_{w}(\sigma(\Lambda)) \quad \text { respectively } \quad V_{\sigma w}(\Lambda)=V_{\sigma w \sigma^{-1}}(\sigma(\Lambda)) .
$$

Recall that for a simple root $\alpha$ the Demazure module $V_{w \sigma}(\Lambda)$ is stable for the associated subalgebra $\mathfrak{s l}_{2}(\alpha)$ if and only if $s_{\alpha} w \sigma \leq w \sigma \bmod W_{\Lambda}$ in the (extended) Bruhat order. In particular, $V_{w \sigma}(\Lambda)$ is a $\mathfrak{g}$-module if and only if $s_{i} w \sigma \leq w \sigma \bmod W_{\Lambda}^{\text {aff }}$ for all $i=1, \ldots, n$.

The example which will interest us are the Demazure modules associated to the weight $r \Lambda_{0}$ for $r \geq 1$, in this case $W_{\Lambda}^{\text {aff }}=W$, so $\widetilde{W}^{\text {aff }} / W=L$. The Demazure module $V_{t_{\nu(\mu \vee} \vee}\left(\Lambda_{0}\right)$ is a $\mathfrak{g}$-module if and only if $\mu^{\vee}$ is an anti-dominant coweight, or, in other words, $\mu^{\vee}=-\lambda^{\vee}$ for some dominant coweight.

To simplify the notation, we write in the following

$$
V_{-\lambda \vee}\left(m \Lambda_{0}\right) \quad \text { for } \quad V_{t_{-\nu(\lambda \vee}}\left(m \Lambda_{0}\right)
$$

and we write

$$
\bar{V}_{-\lambda \vee}\left(m \Lambda_{0}\right),
$$

for $V_{-\lambda \vee}\left(m \Lambda_{0}\right)$ viewed as a $\mathfrak{g}$-module. So we view $\operatorname{Char} \bar{V}_{-\lambda \vee}\left(m \Lambda_{0}\right)$ as an element in $\mathbb{Z}[P]$ obtained from the $\widehat{\mathfrak{h}}$-character by projection.

\subsection{Demazure operators}

Let $\beta$ be a real root of the root system $\widehat{\Phi}$. We define the Demazure operator:

$$
D_{\beta}: \mathbb{Z}[\widehat{P}] \longrightarrow \mathbb{Z}[\widehat{P}], \quad D_{\beta}\left(e^{\lambda}\right)=\frac{e^{\lambda}-e^{s_{\beta}(\lambda)-\beta}}{1-e^{-\beta}}
$$


Lemma 1. 1. For $\lambda, \mu \in \widehat{P}$ we have:

$$
D_{\beta}\left(e^{\lambda}\right)= \begin{cases}e^{\lambda}+e^{\lambda-\beta}+\cdots+e^{s_{\beta}(\lambda)} & \text { if }\left\langle\lambda, \beta^{\vee}\right\rangle \geq 0 \\ 0 & \text { if }\left\langle\lambda, \beta^{\vee}\right\rangle=-1 \\ -e^{\lambda+\beta}-e^{\lambda+2 \beta}-\cdots-e^{s_{\beta}(\lambda)-\beta} & \text { if }\left\langle\lambda, \beta^{\vee}\right\rangle \leq-2\end{cases}
$$

2. $D_{\beta}\left(e^{\lambda+\mu}\right)=e^{\lambda} D_{\beta}\left(e^{\mu}\right)+e^{s_{\beta}(\mu)} D_{\beta}\left(e^{\lambda}\right)-e^{\lambda+s_{\beta}(\mu)}$.

3. Let $\chi \in \mathbb{Z}[\widehat{P}]$ be such that $s_{\beta}(\chi)=\chi$, then $D_{\beta}(\chi)=\chi$.

4. Let $\chi \in \mathbb{Z}[\widehat{P}]$, then $D_{\beta}(\chi)$ is stable under $s_{\beta}$. In particular, if $D_{\beta}(\chi)=$ $\chi$, then $s_{\beta}(\chi)=\chi$.

5. $D_{\beta}$ is idempotent, i.e., $D_{\beta}\left(D_{\beta}\left(e^{\mu}\right)\right)=D_{\beta}\left(e^{\mu}\right)$ for all $\mu$.

Proof. For 1, 3, 4, and 5 see [3, (1.5)-(1.8)]. The proof of part 2 is a simple calculation.

Lemma 1 implies:

Corollary 2. If $\left\langle\mu, \beta^{\vee}\right\rangle=0$, then $D_{\beta}\left(e^{\lambda+\mu}\right)=e^{\mu} D_{\beta}\left(e^{\lambda}\right)$.

The corollary is in fact a special case of the following more general exchange rule, which follows easily from Lemma 1:

Lemma 2. Let $\chi, \eta \in \mathbb{Z}[\widehat{P}]$. If $D_{\beta}(\eta)=\eta$, then

$$
D_{\beta}(\chi \cdot \eta)=\eta \cdot\left(D_{\beta}(\chi)\right)
$$

Since $D_{\alpha_{i}}\left(1-e^{\delta}\right)=\left(1-e^{\delta}\right)$ for all $i=0, \ldots, n$, Lemma 2 shows that the ideal $I_{\delta}$ is stable under all Demazure operators $D_{\beta}$. Thus we obtain induced operators (we still use the same notation $D_{\beta}$ )

$$
D_{\beta}: \mathbb{Z}\left[P_{\mathrm{Sc}}\right] \longrightarrow \mathbb{Z}\left[P_{\mathrm{sc}}\right], \quad e^{\lambda}+I_{\delta} \longmapsto D_{\beta}\left(e^{\lambda}\right)+I_{\delta}
$$

Recall further that $\left\langle\delta, \beta^{\vee}\right\rangle=0$ (see (4)), so it makes sense to define on $\mathbb{Z}\left[P_{\mathrm{sc}}\right]$ the function $e^{\lambda} \mapsto\left\langle\lambda, \beta^{\vee}\right\rangle$.

Lemma 3. If $\lambda \in \widehat{P} \cap \mathfrak{h}^{*}$, then $D_{\alpha_{0}}\left(e^{\lambda}\right)=D_{-\Theta}\left(e^{\lambda}\right)$ in $\mathbb{Z}\left[P_{\mathrm{sc}}\right]$. 
Proof. Since $\lambda \in \mathfrak{h}^{*}$ we have $\left\langle\lambda, \alpha_{0}^{\vee}\right\rangle=\left\langle\lambda, c-\Theta^{\vee}\right\rangle=-\left\langle\lambda, \Theta^{\vee}\right\rangle$. Further, $\alpha_{0}=\delta-\Theta$, so equation (10) can be read in $\mathbb{Z}\left[P_{\mathrm{sc}}\right]$ as

$$
\begin{aligned}
D_{\alpha_{0}}\left(e^{\lambda}\right) & = \begin{cases}e^{\lambda}+e^{\lambda+\Theta}+\cdots+e^{\lambda+n \Theta} & \text { if } n=\left\langle\lambda, \alpha_{0}^{\vee}\right\rangle=\left\langle\lambda,-\Theta^{\vee}\right\rangle \geq 0 \\
0 & \text { if }\left\langle\lambda, \alpha_{0}^{\vee}\right\rangle=\left\langle\lambda,-\Theta^{\vee}\right\rangle=-1 \\
-e^{\lambda-\Theta}-\cdots-e^{\lambda-(|n|-1) \Theta} & \text { if } n=\left\langle\lambda, \alpha_{0}^{\vee}\right\rangle=\left\langle\lambda,-\Theta^{\vee}\right\rangle \leq-2\end{cases} \\
& =D_{-\Theta}\left(e^{\lambda}\right) .
\end{aligned}
$$

\subsection{Demazure character formula}

We want to extend the notion of a Demazure operator also to elements of $\Sigma$. We define for $\sigma \in \Sigma$ :

$$
D_{\sigma}: \mathbb{Z}[\widehat{P}] \longrightarrow \mathbb{Z}[\widehat{P}], \quad D_{\sigma}\left(e^{\Lambda}\right)=e^{\sigma(\Lambda)} .
$$

Since $\sigma(\delta)=\delta$, we get an induced operator $D_{\sigma}$ on $\mathbb{Z}\left[P_{\mathrm{sc}}\right]$.

Lemma 4. $D_{\sigma} D_{\beta}=D_{\sigma(\beta)} D_{\sigma}$.

Proof. Let $\Lambda \in \widehat{P}$, then $\left\langle\Lambda, \beta^{\vee}\right\rangle=\left\langle\sigma(\Lambda), \sigma\left(\beta^{\vee}\right)\right\rangle$, which implies the claim by equation (10).

In the following we denote by $D_{i}, i=0, \ldots, n$ the Demazure operator $D_{\alpha_{i}}$ corresponding to the simple root $\alpha_{i}$. Recall that for any reduced decomposition $w=s_{i_{1}} \cdots s_{i_{r}}$ of $w \in W^{\text {aff }}$ the operator $D_{w}=D_{i_{1}} \cdots D_{i_{r}}$ is independent of the choice of the decomposition (see [11, Corollary 8.2.10]).

We associate an operator to any element $w \sigma \in \widetilde{W}^{\text {aff }}$ by setting

$$
\begin{aligned}
D_{w \sigma}: \mathbb{Z}\left[P_{\mathrm{sc}}\right] & \longrightarrow \mathbb{Z}\left[P_{\mathrm{sc}}\right] \\
e^{\Lambda} & \longmapsto D_{w}\left(e^{\sigma(\Lambda)}\right)
\end{aligned}
$$

By Lemma 4 we have for $\sigma w \in \widetilde{W}^{\text {aff }}=\Sigma \ltimes W^{\text {aff }}$ :

$$
\begin{aligned}
& D_{\sigma w}: \mathbb{Z}\left[P_{\mathrm{Sc}}\right] \longrightarrow \mathbb{Z}\left[P_{\mathrm{sc}}\right] \\
& e^{\Lambda} \longmapsto \sigma\left(D_{w}\left(e^{\Lambda}\right)\right)=D_{\sigma w \sigma^{-1}}\left(e^{\sigma(\Lambda)}\right)
\end{aligned}
$$

Let $w \sigma \in \widetilde{W^{\text {aff }}}$ and let $\Lambda \in \widehat{P}^{+}$be a dominant weight. 
Theorem 1. ([11, Chapter VIII], [12], [17])

$$
\text { Char } V_{w}(\sigma(\Lambda))=D_{w \sigma}\left(e^{\Lambda}\right) .
$$

Let $\lambda^{\vee}$ be a dominant coweight. Associated to $t_{-\nu(\lambda)} \in \widetilde{W}^{\text {aff }}$ we have a Demazure operator $D_{\left.t_{-\nu(\lambda \vee}\right)}$, we write for simplicity just $D_{-\lambda^{\vee}}$.

Lemma 5. Let $\lambda_{1}^{\vee}, \lambda_{2}^{\vee}$ be two dominant coweights, and set $\lambda^{\vee}=\lambda_{1}^{\vee}+$ $\lambda_{2}^{\vee}$. Then

$$
D_{-\lambda_{1}^{\vee}} D_{-\lambda_{2}^{\vee}}=D_{-\lambda^{\vee}} .
$$

Lemma 6. Let $V$ be a finite dimensional $\mathfrak{g}_{\mathrm{sc}}$-module such that Char $V \in$ $\mathbb{Z}[P]$, then

$$
D_{i}(\operatorname{Char} V)=\text { Char } V \quad \forall i=0, \ldots, n \text {; and } D_{\sigma}(\operatorname{Char} V)=\text { Char } V .
$$

Proof. The character of a finite dimensional $\mathfrak{g}$-module is stable under the Weyl group $W$ and hence stable under $D_{i}$ for all $i=1, \ldots, n$ by Lemma 1. It remains to consider the case $i=0$. Now all weights lie in $\mathfrak{h}^{*}$, so by Lemma 3 we have:

$$
D_{0}(\operatorname{Char} V)=D_{-\Theta}(\operatorname{Char} V)=\text { Char } V
$$

where the right hand side is again a consequence of Lemma 1.

Now $\sigma=y t_{-\nu\left(\omega_{j}^{\vee}\right)}$ for some minuscule fundamental coweight $\omega_{j}^{\vee}$ and some $y \in W$. Since $t_{-\nu\left(\omega_{j}^{\vee}\right)}$ operates trivially on $\mathbb{Z}[P]$ and $D_{y}($ Char $V)=$ Char $V$, the claim follows.

\section{$\S 3$. The proofs}

\subsection{Proof of Theorem 1}

Let $\lambda^{\vee}$ be a dominant coweight and suppose we are given a decomposition

$$
\lambda^{\vee}=\lambda_{1}^{\vee}+\lambda_{2}^{\vee}+\cdots+\lambda_{r}^{\vee}
$$

of $\lambda^{\vee}$ as a sum of dominant coweights. For the notation see (8) and (9).

THEOREM 1. As $\mathfrak{g}$-representations, the modules

$$
\bar{V}_{-\lambda \vee}\left(m \Lambda_{0}\right) \quad \text { and } \quad \bar{V}_{-\lambda_{1}^{\vee}}\left(m \Lambda_{0}\right) \otimes \bar{V}_{-\lambda_{2}^{\vee}}\left(m \Lambda_{0}\right) \otimes \cdots \otimes \bar{V}_{-\lambda_{r}^{\vee}}\left(m \Lambda_{0}\right)
$$

are isomorphic. 
More precisely, we will show that, on the level of characters of $\mathfrak{g}_{\mathrm{sc}^{-}}$ modules:

\section{THEOREM $1^{\prime}$.}

Char $V_{-\lambda \vee}\left(m \Lambda_{0}\right)$

$$
=e^{m \Lambda_{0}} \operatorname{Char} \bar{V}_{-\lambda_{1}^{\vee}}\left(m \Lambda_{0}\right) \operatorname{Char} \bar{V}_{-\lambda_{2}^{\vee}}\left(m \Lambda_{0}\right) \cdots \operatorname{Char} \bar{V}_{-\lambda_{r}^{\vee}}\left(m \Lambda_{0}\right) \text {. }
$$

Theorem $1^{\prime}$ obviously implies Theorem 1 , so it suffices to prove Theorem $1^{\prime}$.

A first step is the following lemma:

LEMmA 7. Let $\chi \in \mathbb{Z}\left[P_{\mathrm{sc}}\right]$ be a character of the form $e^{m \Lambda_{0}}$ Char $\bar{V}$, where $\bar{V}$ is a finite dimensional $\mathfrak{g}$-module. Suppose $\lambda^{\vee} \in P^{\vee}$ is a dominant coweight and let $t_{-\nu\left(\lambda^{\vee}\right)}=s_{i_{1}} \cdots s_{i_{t}} \sigma$ be a reduced decomposition in $\widetilde{W}^{\text {aff }}$. Then

$$
D_{i_{1}} \cdots D_{i_{t}} D_{\sigma}\left(e^{m \Lambda_{0}} \operatorname{Char} \bar{V}\right)=D_{i_{1}} \cdots D_{i_{t}} D_{\sigma}\left(e^{m \Lambda_{0}}\right) \text { Char } \bar{V}
$$

Proof. The lemma is proven exactly in the same way as Lemma 2, only using now in addition Lemma 6 for the operators $D_{0}$ and $D_{\sigma}$.

Proof of Theorem 1'. The proof is by induction on $r$. Suppose $r=1$ and $\lambda^{\vee}=w \sigma$ where $\sigma \in \Sigma$ and $w \in W^{\text {aff }}$. The character of $V_{-\lambda^{\vee}}\left(m \Lambda_{0}\right)$ is the character of the Demazure submodule $V_{w}\left(\sigma\left(m \Lambda_{0}\right)\right)=V_{w}\left(m \Lambda_{0}+m \omega_{i}^{*}\right)$ for some appropriate minuscule fundamental weight of $\mathfrak{g}$. So all $\mathfrak{g}_{\mathrm{sc}}$-weights occuring in the module are of the form $m \Lambda_{0}+m \omega_{i}^{*}+$ a sum of roots in $\Phi$ (possibly positive and negative, see Lemma 3 ), and hence the character is of the desired form $e^{m \Lambda_{0}}$ Char $\bar{V}_{-\lambda \vee}\left(m \Lambda_{0}\right)$.

Suppose now $r \geq 2$ and the claim holds already for $r-1$. By the definition in equation $(7)$ we have for $t_{\nu\left(-\lambda^{\vee}\right)}=w \sigma \in \widetilde{W}^{\text {aff }}$ :

$$
\text { Char } V_{-\lambda \vee}\left(m \Lambda_{0}\right)=\operatorname{Char} V_{w}\left(m \sigma\left(\Lambda_{0}\right)\right) \text {, }
$$

by the Demazure character formula (Theorem 1) the latter is equal to $D_{-\lambda \vee}\left(e^{m \Lambda_{0}}\right)$, so

$$
\text { Char } V_{-\lambda \vee}\left(m \Lambda_{0}\right)=D_{-\lambda \vee}\left(e^{m \Lambda_{0}}\right),
$$

by Lemma 5 the right hand side can be rewritten as

$$
\operatorname{Char} V_{-\lambda \vee}\left(m \Lambda_{0}\right)=D_{-\lambda_{1}^{\vee}}\left(D_{-\lambda_{2}^{\vee}} \cdots D_{-\lambda_{r}^{\vee}}\left(e^{m \Lambda_{0}}\right)\right),
$$


by induction the right hand side can be reformulated as

$$
\text { Char } V_{-\lambda \vee}\left(m \Lambda_{0}\right)=D_{-\lambda_{1}^{\vee}}\left(e^{m \Lambda_{0}} \operatorname{Char} \bar{V}_{-\lambda_{2}^{\vee}}\left(m \Lambda_{0}\right) \cdots \operatorname{Char} \bar{V}_{-\lambda_{r}^{\vee}}\left(m \Lambda_{0}\right)\right) \text {, }
$$

by Lemma 7 this is equivalent to

Char $V_{-\lambda \vee}\left(m \Lambda_{0}\right)=\left(D_{-\lambda_{1}^{\vee}}\left(e^{m \Lambda_{0}}\right)\right) \operatorname{Char} \bar{V}_{-\lambda_{2}^{\vee}}\left(m \Lambda_{0}\right) \cdots \operatorname{Char} \bar{V}_{-\lambda_{r}^{\vee}}\left(m \Lambda_{0}\right)$.

Now the arguments for the proof of the case $r=1$ show that this implies

Char $V_{-\lambda \vee}\left(m \Lambda_{0}\right)$

$$
=e^{m \Lambda_{0}} \operatorname{Char} \bar{V}_{-\lambda_{1}^{\vee}}\left(m \Lambda_{0}\right) \operatorname{Char} \bar{V}_{-\lambda_{2}^{\vee}}\left(m \Lambda_{0}\right) \cdots \operatorname{Char} \bar{V}_{-\lambda_{r}^{\vee}}\left(m \Lambda_{0}\right) \text {, }
$$

which finishes the proof.

\subsection{Proof of Theorem $1 \mathrm{~A}$}

The proof is similar to the proof above, so we give just a short sketch. As above, we have

$$
\text { Char } \begin{aligned}
V_{-\lambda \vee}\left(m \Lambda_{0}+r \Lambda_{i}\right) & =D_{-\lambda \vee}\left(e^{m \Lambda_{0}+r \Lambda_{i}}\right) \\
& =D_{-\lambda_{2}^{\vee}} D_{-\lambda_{3}^{\vee}} \cdots D_{-\lambda_{r}} D_{-\omega_{i}^{\vee}}\left(e^{m \Lambda_{0}+r \Lambda_{i}}\right) .
\end{aligned}
$$

Now $t_{-\nu\left(\omega_{i}\right)}=t_{-\omega_{i}}=\tau_{i} \sigma_{i}$. Here $\tau_{i}=w_{0, i} w_{0}$, where $w_{0}$ is the longest element in $W$ and $w_{0, i}$ is the longest word in the stabilizer $W_{\omega_{i}}$ of $\omega_{i}$, and $\sigma_{i}$ is a diagram automorphism. Note that $\sigma_{i}\left(\Lambda_{i}\right)=\tau_{i}^{-1} t_{-\omega_{i}}\left(\Lambda_{0}+\omega_{i}\right)=$ $\tau_{i}^{-1}\left(\Lambda_{0}\right)=\Lambda_{0}$ and

$$
\begin{aligned}
\sigma_{i}\left(\Lambda_{0}\right) & =\tau_{i}^{-1} t_{-\omega_{i}}\left(\Lambda_{0}\right)=\tau_{i}^{-1}\left(\Lambda_{0}-\omega_{i}\right)=\Lambda_{0}+\tau_{i}^{-1}\left(-\omega_{i}\right) \\
& =\Lambda_{0}+w_{0} w_{0, i}\left(-\omega_{i}\right)=\Lambda_{0}+\omega_{i}^{*},
\end{aligned}
$$

where $\omega^{*}$ denotes the highest weight of the irreducible $\mathfrak{g}$-representation $V\left(\omega_{i}\right)^{*}$. Note that $\Lambda_{0}+\omega_{i}^{*}$ is again a fundamental weight (for the KacMoody algebra $\widehat{\mathfrak{g}})$, and recall that

$$
\tau_{i}=w_{0, i} w_{0}=w_{0}\left(w_{0}^{-1} w_{0, i} w_{0}\right)=w_{0} w_{0, i}^{*},
$$

where $w_{0, i}^{*}$ is the longest word in the stabilizer $W_{\omega_{i}^{*}}$ of $\omega_{i}^{*}$. So

$$
\begin{aligned}
D_{-\omega_{i}^{\vee}}\left(e^{m \Lambda_{0}+r \Lambda_{i}}\right) & =D_{\tau_{i}} D_{\sigma_{i}}\left(e^{m \Lambda_{0}+r \Lambda_{i}}\right) \\
& =D_{\tau_{i}}\left(e^{m \Lambda_{0}+m \omega_{i}^{*}+r \Lambda_{0}}\right) \\
& =e^{(m+r) \Lambda_{0}} D_{\tau_{i}}\left(e^{m \omega_{i}^{*}}\right) \\
& =e^{(m+r) \Lambda_{0}} D_{w_{0} w_{0, i}^{*}}\left(e^{m \omega_{i}^{*}}\right) \\
& =e^{(m+r) \Lambda_{0}} \operatorname{Char} V\left(m \omega_{i}^{*}\right) .
\end{aligned}
$$

Now the same induction procedure as above applies to finish the proof. 


\subsection{Proof of Theorem 2}

The proof is divided into several case by case considerations. Suppose first that $\omega^{\vee}$ is a minuscule coweight. In this case (for $m=1$ this has already been proved in [16]) $t_{-\omega_{i}}=w_{i, 0} w_{0} \sigma_{i}$ and hence

$$
\begin{aligned}
\operatorname{Char} \bar{V}_{-\omega \vee}\left(m \Lambda_{0}\right) & =D_{-\omega^{\vee}} e^{m \Lambda_{0}}=D_{w_{i, 0} w_{0}} e^{m \Lambda_{0}+m \omega^{*}}=D_{w_{0} w_{i, 0}^{*}} e^{m \Lambda_{0}+m \omega^{*}} \\
& =e^{m \Lambda_{0}} \operatorname{Char} V(m \omega)^{*} .
\end{aligned}
$$

In particular, this finishes the proof for the Lie algebras of type $A_{n}$. For the next few cases we need the following:

Lemma 8. Let $w_{0}$ be the longest element in the Weyl group of $\mathfrak{g}$, let $z$ be an arbitrary element of $\operatorname{Stab}_{W}(\Theta)$, where $\Theta$ is the highest root of $\mathfrak{g}$, let $r \in \mathbb{N}$. Then

$$
\bar{V}_{w_{0} z s_{0}}\left(r \Lambda_{0}\right) \simeq \bigoplus_{m=0}^{r} V(m \Theta)
$$

as $\mathfrak{g}$-representations.

Proof.

$$
\begin{aligned}
D_{\left(w_{0} z\right) s_{0}}\left(e^{r \Lambda_{0}}\right) & =D_{w_{0} z} D_{-\Theta}\left(e^{r \Lambda_{0}}\right) \\
& =D_{w_{0} z}\left(e^{r \Lambda_{0}}+e^{r \Lambda_{0}+\Theta}+\cdots+e^{r \Lambda_{0}+r \Theta}\right) \\
& =D_{w_{0}}\left(e^{r \Lambda_{0}}+e^{r \Lambda_{0}+\Theta}+\cdots+e^{r \Lambda_{0}+r \Theta}\right) \\
& =e^{r \Lambda_{0}}\left(D_{w_{0}}\left(e^{0}+D_{w_{0}}\left(e^{\Theta}\right)+\cdots+D_{w_{0}}\left(e^{r \Theta}\right)\right)\right)
\end{aligned}
$$

which finishes the proof.

In the cases $E_{6}, E_{7}, E_{8}, F_{4}, G_{2}$ the highest root $\Theta$ is also a fundamental weight, say $\omega_{i}$. Let $p_{i}:=\frac{a_{i}}{a_{i}^{\vee}}$. Then $\nu\left(\omega_{i}^{\vee}\right)=\frac{a_{i}}{a_{i}^{\vee}} \omega_{i}=p_{i} \Theta$. In fact, for the adjoint representations considered here one sees that $p_{i}=1$ in all cases. Since $t_{-\omega_{i}}=s_{\theta} s_{0}$, it follows by Lemma 8:

$$
\bar{V}_{-\omega_{i}^{\vee}}\left(r \Lambda_{0}\right) \simeq \bigoplus_{m=0}^{r} V\left(m \omega_{i}\right) .
$$

Next we consider the types $\mathrm{B}_{n}$ and $\mathrm{D}_{n}$ with the Bourbaki indexing of the simple roots, i.e., we consider the root system as embedded in $\mathbb{R}^{n}$ with the canonical basis $\left\{\epsilon_{1}, \ldots, \epsilon_{n}\right\}$ and the standard scalar product. The basis of 
the root system is given by the simple roots $\alpha_{i}=\epsilon_{i}-\epsilon_{i+1}, i=1, \ldots, n-1$ and $\alpha_{n}=\epsilon_{n}$ (type $\mathrm{B}_{n}, n \geq 3$ ) respectively $\alpha_{n}=\epsilon_{n-1}+\epsilon_{n}$ (type $\mathrm{D}_{n}, n \geq 4$ ), the highest root is $\epsilon_{1}+\epsilon_{2}$ in both cases. We have

$$
\begin{aligned}
t_{-\omega_{2}} & =s_{\epsilon_{1}+\epsilon_{2}} s_{0} \\
& =\left(s_{2} \cdots s_{n} \cdots s_{2}\right) s_{1}\left(s_{2} \cdots s_{n} \cdots s_{2}\right) s_{0} .
\end{aligned}
$$

In the following we consider only the non-minuscule fundamental coweights. We get for $2 i \leq n\left(\right.$ case $\left.\mathrm{B}_{n}\right)$ respectively $2 i \leq n-2\left(\right.$ case $\left.\mathrm{D}_{n}\right)$ :

$$
\begin{aligned}
t_{-\nu\left(\omega_{2 i}^{\vee}\right)=} & t_{-\epsilon_{1}-\epsilon_{2}} t_{-\epsilon_{3}-\epsilon_{4}} \cdots t_{-\epsilon_{2 i-1}-\epsilon_{2 i}} \\
= & t_{-\omega_{2}}\left(\left(s_{2} s_{1} s_{3} s_{2}\right) t_{-\omega_{2}}\left(s_{2} s_{1} s_{3} s_{2}\right)\right) \cdots\left(\left(s_{2 i-2} \cdots s_{2} s_{1}\right)\right. \\
& \left.\quad\left(s_{2 i-1} \cdots s_{3} s_{2}\right) t_{-\omega_{2}}\left(s_{2} s_{3} \cdots s_{2 i-1}\right)\left(s_{1} s_{2} \cdots s_{2 i-2}\right)\right) \\
= & s_{\epsilon_{1}+\epsilon_{2}} s_{0} s_{\epsilon_{3}+\epsilon_{4}}\left(\left(s_{2} s_{1} s_{3} s_{2}\right) s_{0}\left(s_{2} s_{1} s_{3} s_{2}\right)\right) s_{\epsilon_{5}+\epsilon_{6}} \cdots s_{\epsilon_{2 i-1}+\epsilon_{2 i}} \\
& \quad\left(\left(s_{2 i-2} \cdots s_{2} s_{1}\right)\left(s_{2 i-1} \cdots s_{3} s_{2}\right) s_{0}\left(s_{2} s_{3} \cdots s_{2 i-1}\right)\left(s_{1} s_{2} \cdots s_{2 i-2}\right)\right) \\
= & {\left[s_{\epsilon_{1}+\epsilon_{2}} s_{\epsilon_{3}+\epsilon_{4}} \cdots s_{\epsilon_{2 i-1}+\epsilon_{2 i}}\right]\left[s_{0}\left(\left(s_{2} s_{1} s_{3} s_{2}\right) s_{0}\left(s_{2} s_{1} s_{3} s_{2}\right)\right) \cdots\right.} \\
& \left.\quad\left(\left(s_{2 i-2} \cdots s_{2} s_{1}\right)\left(s_{2 i-1} \cdots s_{3} s_{2}\right) s_{0}\left(s_{2} s_{3} \cdots s_{2 i-1}\right)\left(s_{1} s_{2} \cdots s_{2 i-2}\right)\right)\right] .
\end{aligned}
$$

We see that we can write the word as a product $w_{1} w_{2}$ of two words, the first being an element of the Weyl group $W$ and the second being a word in the subgroup of $W^{\text {aff }}$ generated by the simple reflections $s_{0}, s_{1}, \ldots, s_{2 i-1}$, this is (in the $\mathrm{B}_{n}$ as well as in the $\mathrm{D}_{n}$ case) a group of type $\mathrm{D}_{2 i}$.

Since we look for a character of a $\mathfrak{g}$-module, we know the character is stable under the operators $D_{i}, 1 \leq i \leq n$. So to determine the character of $V_{-\omega_{2 i}}\left(m \Lambda_{0}\right)$, it suffices to get a reduced decomposition of the word $w_{2}$ above modulo the right and left action of $W$, the character of $V_{-\omega_{2 i}}\left(m \Lambda_{0}\right)$ can be reconstructed by applying the Demazure operators $D_{i}, 1 \leq i \leq n$.

The strategy is the following. We show that the decomposition above of $w_{2}$ is a reduced decomposition. Further, we show that $\tau=s_{1} s_{3} \cdots s_{2 i-1} w_{2}$ is the longest word of the Weyl group of the subgroup of $W^{\text {aff }}$ of type $\mathrm{D}_{2 i}$.

Before we give a more detailed account on how to prove this, let us show how this solves the problem. Let $\mathfrak{d} \subset \widehat{\mathfrak{g}}$ be the semisimple Lie algebra of type $\mathrm{D}_{2 i}$ associated to the simple roots $\alpha_{0}, \ldots, \alpha_{2 i-1}$, then $V_{\tau}\left(m \Lambda_{0}\right)$ is an irreducible $\mathfrak{d}$-module. More precisely, it is the irreducible $m$-th spin representation (associated to the node of $\alpha_{0}$ ). Let $\mathfrak{d}^{\prime}$ be the semisimple 
subalgebra of $\mathfrak{d}$ corresponding to the simple roots $\alpha_{1}, \ldots, \alpha_{2 i-1}$, then $\mathfrak{d}^{\prime}$ is also the semisimple part of a Levi subalgebra of $\mathfrak{g}$. Since $V_{\tau}\left(m \Lambda_{0}\right)$ is a $\widehat{\mathfrak{b}}$-module, it is hence a $\mathfrak{b}$ and a $\mathfrak{d}^{\prime}$-module. By the Borel-Weil-Bott theorem we know that the induced $\mathfrak{g}$-module (which is the module $V_{-\omega_{2 i}^{\vee}}\left(m \Lambda_{0}\right)$ ) has the same direct sum decomposition as $V_{\tau}\left(m \Lambda_{0}\right)$ has as $\mathfrak{d}^{\prime}$-module. Since the latter has been already given in [15], this finishes the proof.

We come now back to the proof of the first claim. We make the calculations in the following modulo $\delta$, so the set of positive roots for the type $\mathrm{D}_{2 i}$-subdiagram (modulo $\delta$ ) is the set

$$
\left\{\epsilon_{s}-\epsilon_{t} \mid 1 \leq s<t \leq 2 i\right\} \cup\left\{-\epsilon_{s}-\epsilon_{t} \mid 1 \leq s<t \leq 2 i\right\} .
$$

In these terms the decomposition of $w_{2}$ as the second part in the square brackets in (13), reads as

$$
w_{2}=s_{-\epsilon_{1}-\epsilon_{2}} s_{-\epsilon_{3}-\epsilon_{4}} \cdots s_{-\epsilon_{2 i-1}-\epsilon_{2 i}} .
$$

and all positive roots above are sent to negative by $w_{2}$ roots except $\alpha_{1}, \alpha_{3}$, $\ldots, \alpha_{2 i-1}$. This implies that the decomposition above of length $4 i^{2}-3 i$ is reduced, and

$$
\begin{aligned}
& \tau=s_{1} s_{3} \ldots s_{2 i-1} s_{0}\left(\left(s_{2} s_{1} s_{3} s_{2}\right) s_{0}\left(s_{2} s_{1} s_{3} s_{2}\right)\right) \cdots \\
& \quad\left(\left(s_{2 i-2} \cdots s_{2} s_{1}\right)\left(s_{2 i-1} \cdots s_{3} s_{2}\right) s_{0}\left(s_{2} s_{3} \cdots s_{2 i-1}\right)\left(s_{1} s_{2} \cdots s_{2 i-2}\right)\right)
\end{aligned}
$$

is a reduced decomposition of the longest word of the Weyl group of the subgroup of type $\mathrm{D}_{2 i}$. This shows that $\tau$ a subword of $t_{-\omega_{2 i}}$, and $V_{-\omega_{2 i}^{\vee}}\left(m \Lambda_{0}\right)$ is the $\mathfrak{g}$-module generated by the $\mathfrak{b}$ - $\mathfrak{d}^{\prime}$-submodule $V_{\tau}\left(m \Lambda_{0}\right)$.

It has been already pointed out above that the decomposition of $V_{-\omega_{2 i}^{\vee}}\left(m \Lambda_{0}\right)$ as $\mathfrak{g}$-module is completely determined by the $\mathfrak{h}$-module structure of $V_{\tau}\left(m \Lambda_{0}\right)$ and the decomposition of $V_{\tau}\left(m \Lambda_{0}\right)$ as $\mathfrak{d}^{\prime}$-module. So it remains to describe the decomposition of the $m$-th spin-representation $V_{\tau}\left(m \Lambda_{0}\right)$ with respect to the subalgebra $\mathfrak{d}^{\prime}$, and to describe the highest weights as weights for the Cartan subalgebra $\mathfrak{h}$.

The decomposition of the $m$-th spin-representation $V_{\tau}\left(m \Lambda_{0}\right)$ with respect to the subalgebra $\mathfrak{d}^{\prime}$ can be found in [15] (see Section 1.4). The description of the possible highest weights occuring ([15, Proposition 3.2]) in the decomposition implies for $2 i<n$ (case $\mathrm{B}_{n}$ ) respectively $2 i \leq n-2$ (case $\mathrm{D}_{n}$ ):

$$
\operatorname{Char} \bar{V}_{-\omega_{2 i}^{\vee}}\left(m \Lambda_{0}\right)=\sum_{a_{1}+\cdots+a_{i}=m} \operatorname{Char} V\left(a_{1} \omega_{2}+\cdots+a_{i} \omega_{2 i}\right),
$$


and for $2 i=n$ in the case $\mathrm{B}_{n}$ :

$\operatorname{Char} \bar{V}_{-\omega_{n}^{\vee}}\left(m \Lambda_{0}\right)=\sum_{a_{1}+\cdots+a_{n / 2}=m} \operatorname{Char} V\left(a_{1} \omega_{2}+\cdots+a_{(n-2) / 2} \omega_{n-2}+2 a_{n} \omega_{n}\right)$.

The calculation for the odd case is similar. We assume $2 i+1 \leq n$ in the case $\mathrm{B}_{n}$ and $2 i+1 \leq n-2$ in the case $\mathrm{D}_{n}$

$$
\begin{aligned}
& t_{-\omega_{2 i+1}^{\vee}}=t_{-\epsilon_{1}-\epsilon_{2}} \cdots t_{-\epsilon_{2 i-1}-\epsilon_{2 i}} t_{-\epsilon_{2 i+1}} \\
& =t_{-\epsilon_{1}-\epsilon_{2}}\left(s_{2} s_{1} s_{3} s_{2} t_{-\epsilon_{1}-\epsilon_{2}} s_{2} s_{3} s_{1} s_{2}\right) \cdots \\
& \left(s_{2 i-2} \cdots s_{1} s_{2 i-1} \cdots s_{2} t_{-\epsilon_{1}-\epsilon_{2}} s_{2} \cdots s_{2 i-1} s_{1} \cdots s_{2 i-2}\right) \\
& \left(s_{2 i} \cdots s_{1} t_{-\epsilon_{1}} s_{1} \cdots s_{2 i}\right) \\
& =s_{\epsilon_{1}+\epsilon_{2}} s_{0} s_{\epsilon_{3}+\epsilon_{4}}\left(s_{2} s_{1} s_{3} s_{2} s_{0} s_{2} s_{3} s_{1} s_{2}\right) \cdots \\
& s_{\epsilon_{2 i-1}+\epsilon_{2 i}}\left(s_{2 i-2} \cdots s_{1} s_{2 i-1} \cdots s_{2} s_{0} s_{2} \cdots s_{2 i-1} s_{1} \cdots s_{2 i-2}\right) \\
& s_{\epsilon_{2 i+1}}\left(s_{2 i} \cdots s_{1} \sigma_{1} s_{1} \cdots s_{2 i}\right) \\
& =\left[s_{\epsilon_{1}+\epsilon_{2}} s_{\epsilon_{3}+\epsilon_{4}} \cdots s_{\epsilon_{2 i-1}+\epsilon_{2 i}} s_{\epsilon_{2 i+1}}\right]\left[s_{0}\left(s_{2} s_{1} s_{3} s_{2} s_{0} s_{2} s_{3} s_{1} s_{2}\right) \cdots\right. \\
& \left(s_{2 i-2} \cdots s_{1} s_{2 i-1} \cdots s_{2} s_{0} s_{2} \cdots s_{2 i-1} s_{1} \cdots s_{2 i-2}\right) \\
& \left.\left(s_{2 i} \cdots s_{1} s_{0} s_{2} \cdots s_{2 i}\right) \sigma_{1}\right] \text {. }
\end{aligned}
$$

It follows as above that the second part of the word is reduced. In fact, after multiplying the word with $s_{1} s_{3} \cdots s_{2 i-1}$, we obtain a reduced decomposition of the longest word

$$
\begin{aligned}
\tau=s_{1} s_{3} \cdots s_{2 i-1} s_{0}\left(s_{2} s_{1} s_{3} s_{2} s_{0} s_{2} s_{3} s_{1} s_{2}\right) \cdots \\
\quad\left(s_{2 i-2} \cdots s_{1} s_{2 i-1} \cdots s_{2} s_{0} s_{2} \cdots s_{2 i-1} s_{1} \cdots s_{2 i-2}\right)\left(s_{2 i} \cdots s_{1} s_{0} s_{2} \cdots s_{2 i}\right)
\end{aligned}
$$

in the Weyl group of the semisimple Lie algebra $\mathfrak{d} \subset \widehat{\mathfrak{g}}$ of type $\mathrm{D}_{2 i+1}$ associated to the simple roots $\alpha_{0}, \ldots, \alpha_{2 i}$. The Demazure module $V_{\tau \sigma_{1}}\left(m \Lambda_{0}\right)$ is an irreducible $\mathfrak{d}$-module, it is the $m$-th spin representation, associated to the node corresponding to $\alpha_{1}$. Consider the decomposition of $V_{\tau \sigma_{1}}\left(m \Lambda_{0}\right)$ as an $\mathfrak{h}$ - and a $\mathfrak{d}^{\prime}$-module, where $\mathfrak{d}^{\prime} \subset \mathfrak{d}$ is the semisimple Lie subalgebra associated to the simple roots $\alpha_{1}, \ldots, \alpha_{2 i}$. By [15], we get as $\mathfrak{d}^{\prime}-\mathfrak{h}$-module the decomposition $\left(2 i+1<n\right.$ in the $\mathrm{B}_{n}$ case):

$$
\bar{V}_{\tau \sigma_{1}}\left(m \Lambda_{0}\right)=\bar{V}_{\tau}\left(m \Lambda_{1}\right)=\bigoplus_{a_{1}+\cdots+a_{i}=m} V\left(a_{1} \omega_{1}+a_{1} \omega_{3}+\cdots+a_{i} \omega_{2 i+1}\right)
$$

and, again by the Borel-Weil-Bott theorem, the same decomposition holds for the Demazure module $V_{-\omega_{2 i+1}^{\vee}}\left(m \Lambda_{0}\right)$ as $\mathfrak{g}$-module. The case $n=2 i+1$ is treated similarly. 
Next we consider the Lie algebra of type $\mathrm{C}_{n}$. We have for $j=1, \ldots, n-1$ $\left(\omega_{n}^{\vee}\right.$ is minuscule)

$$
\begin{aligned}
t_{-\omega_{j}^{\vee}} & =t_{-2 \omega_{j}}=t_{-2 \epsilon_{1}} t_{-2 \epsilon_{2}} \cdots t_{-2 \epsilon_{j}} \\
& =t_{-2 \epsilon_{1}}\left(s_{1} t_{-2 \epsilon_{1}} s_{1}\right) \cdots\left(s_{j-1} \cdots s_{1} t_{-2 \epsilon_{1}} s_{1} \cdots s_{j-1}\right) .
\end{aligned}
$$

Replacing $t_{-2 \epsilon_{1}}$ by $s_{2 \epsilon_{1}} s_{0}$ we get

$$
\begin{aligned}
t_{-\omega_{j}^{\vee}}= & s_{2 \epsilon_{1}} s_{0}\left(s_{1} s_{2 \epsilon_{1}} s_{0} s_{1}\right)\left(s_{2} s_{1} s_{2 \epsilon_{1}} s_{0} s_{1} s_{2}\right) \cdots\left(s_{j-1} \cdots s_{1} s_{2 \epsilon_{1}} s_{0} s_{1} \cdots s_{j-1}\right) \\
= & s_{2 \epsilon_{1}} s_{0} s_{2 \epsilon_{2}}\left(s_{1} s_{0} s_{1}\right) s_{2 \epsilon_{3}}\left(s_{2} s_{1} s_{0} s_{1} s_{2}\right) \cdots s_{2 \epsilon_{j}}\left(s_{j-1} \cdots s_{1} s_{0} s_{1} \cdots s_{j-1}\right) \\
= & {\left[s_{2 \epsilon_{1}} s_{2 \epsilon_{2}} s_{2 \epsilon_{3}} \cdots s_{2 \epsilon_{j}}\right]\left[s_{0}\left(s_{1} s_{0} s_{1}\right)\left(s_{2} s_{1} s_{0} s_{1} s_{2}\right) \cdots\right.} \\
& \left.\left(s_{j-1} \cdots s_{1} s_{0} s_{1} \cdots s_{j-1}\right)\right] .
\end{aligned}
$$

We proceed now with the same strategy as before. For the moment we omit the reflections $s_{2 \epsilon_{1}} s_{2 \epsilon_{2}} s_{2 \epsilon_{3}} \cdots s_{2 \epsilon_{j}}$. The second part, the word

$$
\tau=s_{0}\left(s_{1} s_{0} s_{1}\right)\left(s_{2} s_{1} s_{0} s_{1} s_{2}\right) \cdots\left(s_{j-1} \cdots s_{1} s_{0} s_{1} \cdots s_{j-1}\right),
$$

is a reduced decomposition of the longest word of the semisimple subalgebra $\mathfrak{d} \subset \widehat{\mathfrak{g}}$ of type $\mathrm{C}_{j}$ associated to the simple roots $\alpha_{0}, \ldots, \alpha_{j-1}$.

The Demazure module $V_{\tau}\left(m \Lambda_{0}\right)$ is, as $\mathfrak{d}$-module, irreducible. Let $\mathfrak{d}^{\prime} \subset \mathfrak{d}$ be the semisimple Lie algebra associated to the simple roots $\alpha_{1}, \ldots, \alpha_{j-1}$, it follows again from [15] that the restriction of $V_{\tau}\left(m \Lambda_{0}\right)$ decomposes as $\mathfrak{d}^{\prime}$ and $\mathfrak{h}$-module

$$
\bar{V}_{\tau}\left(m \Lambda_{0}\right) \simeq \bigoplus_{a_{1}+\cdots+a_{j} \leq m} \bar{V}\left(2 a_{1} \omega_{1}+\cdots+2 a_{j} \omega_{j}\right),
$$

which, as above, implies the corresponding decomposition as $\mathfrak{g}$-module.

Fot $\mathfrak{g}$ of type $\mathrm{F}_{4}$ and $\omega_{4}^{\vee}$ we use the same strategy as above. Using the same notation as in [1], one sees $2 \omega_{4}=2 \epsilon_{1}=\left(\epsilon_{1}+\epsilon_{2}\right)+\left(\epsilon_{1}-\epsilon_{2}\right)=$ $\Theta+s_{1} s_{2} s_{3} s_{2} s_{1}(\Theta)$, so

$$
\begin{aligned}
t_{-\nu\left(\omega_{4}^{\vee}\right)} & =t_{-2 \omega_{4}}=t_{-\epsilon_{1}-\epsilon_{2}} t_{-\epsilon_{1}+\epsilon_{2}} \\
& =\left(s_{\Theta} s_{0}\right)\left(s_{1} s_{2} s_{3} s_{2} s_{1} s_{\Theta} s_{1} s_{2} s_{3} s_{2} s_{1}\right) \\
& =\left(s_{\Theta} s_{\epsilon_{1}-\epsilon_{2}}\right)\left(s_{0} s_{1} s_{2} s_{3} s_{2} s_{1} s_{0} s_{1} s_{2} s_{3} s_{2} s_{1}\right) .
\end{aligned}
$$

Again we decompose the translation into a product of two words $w \tau$ such that $w \in W$ and $\tau$ is a subword of a reduced decomposition of the longest 
word of the Weyl group of type $\mathrm{B}_{4}$ corresponding to the roots $\left\{\alpha_{0}, \alpha_{1}, \alpha_{2}, \alpha_{3}\right\}$. For the corresponding Levi subalgebra of $\widehat{\mathfrak{g}}$ we have $V_{\tau}\left(m \Lambda_{0}\right)$ is the Cartan component in the $m$-th symmetric power of the action of the orthogonal Lie algebra on $\mathbb{C}^{n}$. Now by looking at the decomposition of this space with respect to the Levi subalgebra of $\mathfrak{g}$ corresponding to the simple roots $\left\{\alpha_{1}, \alpha_{2}, \alpha_{3}\right\}$ (using again the tables in [15]), we obtain the desired formula.

\subsection{Proof of Theorem 3}

We will need the following simple:

Lemma 9. Let $\lambda^{\vee}$ be a dominant, integral coweight of $\mathfrak{g}$, let $w_{0}$ be the longest element of the Weyl group of $\mathfrak{g}$, then:

$$
l\left(t_{-\lambda \vee} w_{0}\right)=l\left(t_{-\lambda \vee}\right)+l\left(w_{0}\right) .
$$

So reduced decompositions of $t_{-\lambda} \vee$ and $w_{0}$ give a reduced decomposition of $t_{-\lambda} \vee w_{0}$.

LEMma 10. Let $W$ be the $\mathfrak{g}$-module $W:=\bar{V}_{-\theta^{\vee}}\left(r \Lambda_{0}\right)$, then there exists a unique one-dimensional submodule in $W$.

Proof. The proof is by case by case consideration.

- For type $\mathrm{A}_{n}$ we have $\theta^{\vee}=\omega_{1}^{\vee}+\omega_{n}^{\vee}$, so by Theorem 2

$$
\bar{V}_{-\theta^{\vee}}\left(r \Lambda_{0}\right) \simeq V\left(r \omega_{1}^{*}\right) \otimes V\left(r \omega_{n}^{*}\right)
$$

contains an unique one-dimensional submodule.

- For type $\mathrm{B}_{n}$ and $\mathrm{D}_{n}, \theta^{\vee}=\omega_{2}^{\vee}$. By Theorem $2 \bar{V}_{-w_{2}^{\vee}}\left(r \Lambda_{0}\right)$ contains a unique one-dimensional submodule.

- For type $\mathrm{C}_{n}, \theta^{\vee}=\omega_{1}^{\vee}$ and $\theta=2 \omega_{1}$, so again by Theorem $2 \bar{V}_{-\theta^{\vee}}\left(r \Lambda_{0}\right)$ contains a unique one-dimensional submodule.

- If $\mathfrak{g}$ is of type $\mathrm{E}_{6}, \mathrm{E}_{7}, \mathrm{E}_{8}, \mathrm{~F}_{4}, \mathrm{G}_{2}$, then $\theta^{\vee}=\omega_{2}^{\vee}, \omega_{1}^{\vee}, \omega_{8}^{\vee}, \omega_{1}^{\vee}, \omega_{2}^{\vee}$ respectively and the claim follows again by Theorem 2 .

We come to the proof of the theorem:

Proof. Let $\mathrm{W}$ be the g-module $\bar{V}_{s_{\theta} s_{0}}\left(r \Lambda_{0}\right)$. Consider the following sequence of Weyl group elements:

$$
w_{0}<s_{\theta} s_{0} w_{0}<\left(s_{\theta} s_{0}\right)^{2} w_{0}<\left(s_{\theta} s_{0}\right)^{3} w_{0}<\cdots .
$$


Note that the length is additive (recall $t_{-\theta}=s_{\theta} s_{0}$ and Lemma 9), and in a reduced decomposition of $s_{\theta}$ every simple reflection $s_{i}, i=1, \ldots, n$, has to occur. So given an arbitrary element $\kappa \in W^{\text {aff }}$, there exists an $N \in \mathbb{N}$ such that $w \leq\left(s_{\theta} s_{0}\right)^{N} w_{0}$. Hence:

$$
V(\Lambda)=\lim _{N \rightarrow \infty} V_{\left(s_{\theta} s_{0}\right)^{N} w_{0}}(\Lambda)
$$

Write $\Lambda=r \Lambda_{0}+\lambda$, then we obtain (using the Demazure operator)

$$
\begin{aligned}
D_{\left(s_{\theta} s_{0}\right)^{N} w_{0}}\left(e^{\left(r \Lambda_{0}+\lambda\right)}\right) & =D_{\left(s_{\theta} s_{0}\right)^{N}} D_{w_{0}}\left(e^{\left(r \Lambda_{0}+\lambda\right)}\right) \\
& =D_{\left(s_{\theta} s_{0}\right)^{N}}\left(e^{r \Lambda_{0}} \operatorname{Char} V(\lambda)\right) \\
& =e^{r \Lambda_{0}}(\operatorname{Char} W)^{N} \operatorname{Char} V(\lambda) .
\end{aligned}
$$

This shows that in the sequence of inclusions

$$
V(\lambda) \hookrightarrow W \otimes V(\lambda) \hookrightarrow W \otimes W \otimes V(\lambda) \hookrightarrow \cdots
$$

the submodules $W^{\otimes N} \otimes V(\lambda)$ are, as $\mathfrak{g}$-modules, isomorphic to $\bar{V}_{\left(s_{\theta} s_{0}\right)^{N} w_{0}}(\Lambda)$. Now the same arguments as in [16, Chapter 3], prove the theorem.

\section{$\S 4$. The twisted case}

In this section we would like to extend the results to twisted affine Kac-Moody algebras and by the way to so called special vertices. Let $\mathrm{X}_{n}^{(r)}$ be Dynkin diagram of affine type, $r$ the order of the automorphism, in this section we consider only $r>1$. A vertex $k$ of the Dynkin diagram is called special if $\delta-a_{k} \alpha_{k}$ is a positive root, here $\delta, a_{k}, \alpha_{k}$ and so on are defined in the same way as in Chapter 2. For example, 0 is always special vertex, one has $a_{0}=2$ for $\mathrm{A}_{2 l}^{(2)}$ and $a_{0}=1$ for the other case.

Suppose $k$ is a special vertex. Set $\theta_{k}=\delta-a_{k} \alpha_{k}$, we have the finite Weyl group $W_{k}=\left\langle s_{i} \mid i \neq k\right\rangle$ and let $M_{k}$ be the $\mathbb{Z}$-lattice spanned by $\nu\left(W_{k}\left(\theta_{k}^{\vee}\right)\right)$ (see [7] for more details). One knows $([7])$ that the affine Weyl group of $\mathrm{X}_{n}^{(r)}$ is isomorphic to $W_{k} \ltimes t_{M_{k}}$, the semi-direct product of $W_{k}$ with the translations (modulo $\delta$ ) by $M_{k}$. The following Lemma holds:

LEMMA 11. Let $k$ be a special vertex, then $s_{k} s_{\theta_{k}}=t_{\nu\left(\beta^{\vee}\right)}$ modulo $\delta$. For $\lambda$ with $\langle\lambda, K\rangle=0$ it follows:

$$
s_{k} s_{\theta_{k}}(\lambda)=\lambda
$$


Proof.

$$
\begin{aligned}
s_{k} s_{\theta_{k}}(\lambda) & =s_{k}\left(\lambda-\lambda\left(\theta_{k}^{\vee}\right) \theta_{k}\right) \\
& =s_{k}\left(\lambda-\lambda\left(\theta_{k}^{\vee}\right)\left(\delta-a_{k} \alpha_{k}\right)\right) \\
& =\lambda-\left(\lambda\left(\alpha_{k}^{\vee}\right)+a_{k} \lambda\left(\theta_{k}^{\vee}\right)\right) \alpha_{k}-\lambda\left(\theta_{k}^{\vee}\right) \delta .
\end{aligned}
$$

So the lemma follows, because $\lambda\left(\alpha_{k}^{\vee}+a_{k} \theta_{k}^{\vee}\right)=0$, since $\lambda(K)=0$.

In Section 2 we have defined the Demazure operator $D_{\beta}$ for every real root $\beta$, with Lemma 1 and Lemma 11 it follows:

Lemma 12. Let $\chi \in \mathbb{Z}\left[\widehat{P} \cap \mathfrak{h}^{*}\right]$. If $s_{\theta_{k}}(\chi)=\chi$, then $D_{\alpha_{k}}(\chi)=\chi$.

If one deletes in the Dynkin diagram of $\mathrm{X}_{n}^{(r)}$ the zero node, then one gets the diagram (let us call it $\mathrm{Y}_{n}$ ) of a simple Lie Algebra. The following list shows which diagram one gets after removing the zero node, and further, it shows that the positive root $\delta-a_{0} \alpha_{0}$ is a root of $\mathrm{Y}_{n}$.

- for $\mathrm{A}_{2}^{(2)}: \mathrm{A}_{1}$ and $\delta-a_{0} \alpha_{0}=\alpha_{1}=\theta$, the highest root of $\mathrm{A}_{1}$

- for $\mathrm{A}_{2 l}^{(2)}: \mathrm{C}_{l}$ and $\delta-a_{0} \alpha_{0}=\theta^{l}$, the highest long root of $\mathrm{C}_{l}$

- for $\mathrm{A}_{2 l-1}^{(2)}: \mathrm{C}_{l}$ and $\delta-a_{0} \alpha_{0}=\theta^{s}$, the highest short root of $\mathrm{C}_{l}$

- for $\mathrm{D}_{l+1}^{(2)}: \mathrm{B}_{l}$ and $\delta-a_{0} \alpha_{0}=\theta^{s}$, the highest short root of $\mathrm{B}_{l}$

- for $\mathrm{E}_{6}^{(2)}: \mathrm{F}_{4}$ and $\delta-a_{0} \alpha_{0}=\theta^{s}$, the highest short root of $\mathrm{F}_{4}$

- for $\mathrm{D}_{4}^{(3)}: \mathrm{G}_{2}$ and $\delta-a_{0} \alpha_{0}=\theta^{s}$, the highest short root of $\mathrm{G}_{2}$

More generally, a vertex $k$ is special if and only if there exists an automorphism $\sigma$ of the Dynkin diagram, such that $\sigma(k)=0$. In the untwisted case special is the same as minuscule. In the twisted case, there are only for $\mathrm{A}_{2 l-1}^{(2)}$ and $\mathrm{D}_{l+1}^{(2)}$ nontrivial automorphisms. We make a new list now for the twisted case, we delete a special vertex $k \neq 0$.

- for $\mathrm{A}_{2 l-1}^{(2)}$ deleting 1: $\mathrm{C}_{l}$ and $\delta-a_{1} \alpha_{1}=\theta_{1}^{s}$, the highest short root of $\mathrm{C}_{l}$ - for $\mathrm{D}_{l+1}^{(2)}$ deleting $l: \mathrm{B}_{l}$ and $\delta-a_{l} \alpha_{l}=\theta_{l}^{s}$, the highest short root of $\mathrm{B}_{l}$

We get an analog of Lemma 6 . Let $\widehat{\mathfrak{g}}$ be the affine Kac-Moody algebra associated to $\mathrm{X}_{n}^{(r)}$, let $\mathfrak{a}$ be the simple Lie algebra associated to $\mathrm{Y}_{n}$ and denote $P$ the weight lattice of $\mathfrak{a}$. 
LEMMA 13. Let $V$ be a finite dimensional $\mathfrak{a}$ module such that Char $V \in$ $\mathbb{Z}[P]$, then

$$
D_{i}(\operatorname{Char} V)=\text { Char } V \quad \forall i=0, \ldots, n \text {. }
$$

Proof. Char $V$ is stable under $D_{i}, i \geq 1$. In fact, Char $V$ is stable under $D_{\beta}$ for all roots of the Lie algebra $\mathfrak{a}$. So only the case $i=0$ has to be considered. Now all weights in $V$ are of level 0 , so $D_{0}=D_{-a_{0} \theta_{0}}$ $\theta_{0}=\delta-a_{0} \alpha_{0}$, on these weights, which finishes the proof. This suffices to prove this, because if $\chi$ is stable under $D_{\beta}$, then it is stable under $D_{n \beta}$, even if it is not a root.

Recall $P$ is the $\mathbb{Z}$-lattice spanned by the fundamental weights of $\mathfrak{a}$. One can now formulate a statement analogous to Theorem 1. Let $\lambda^{\vee}$ be a dominant element of $M_{k} \subset P_{k}$, where $P_{k}$ are the integral, dominant weights of a. Let $\lambda^{\vee}=\lambda_{1}^{\vee}+\lambda_{2}^{\vee}+\cdots+\lambda_{r}^{\vee}$ be a decomposition of $\lambda^{\vee}$ as a sum of dominant elements of $M_{k}$.

THEOREM 2. Let $k$ be a special vertex of a twisted affine Kac-Moody algebra of type $\mathrm{x}_{n}^{(r)}$, and let $\mathfrak{a}, \ldots$ be as above. For all $m \geq 1$, we have an isomorphism of a-modules between the Demazure module $\bar{V}_{-\lambda} \vee\left(m \Lambda_{k}\right)$ and the tensor product of Demazure modules:

$$
\bar{V}_{-\lambda^{\vee}}\left(m \Lambda_{k}\right) \simeq \bar{V}_{-\lambda_{1}^{\vee}}\left(m \Lambda_{k}\right) \otimes \bar{V}_{-\lambda_{2}^{\vee}}\left(m \Lambda_{k}\right) \otimes \cdots \otimes \bar{V}_{-\lambda_{r}^{\vee}}\left(m \Lambda_{k}\right)
$$

With the Lemma above, the proof is the same as in the untwisted case. As in the untwisted case we can now look in more detail at the smallest Demazure modules $V_{-\omega_{i}}\left(l \Lambda_{0}\right)$, where $\omega_{i}$ is a fundamental weight for $\mathfrak{a}$. The decompositions listed below have been partially calculated (or conjectured) in [4], the remaining cases (and the proofs of the conjectured decompositions) have been calculated by Naito and Sagaki (unpublished result) as in [19]. With a bar we denote again the $\mathfrak{a}$-module, where $\mathfrak{a}$ denotes the simple Lie algebra associated to diagram obtained after removing the zero node. Let $\epsilon=1$ for $i$ odd and 0 for $i$ even.

- $\mathrm{A}_{2 n}^{(2)}, \mathfrak{a}$ is of type $\mathrm{C}_{n}$

$$
\overline{V_{-\omega_{i}}\left(l \Lambda_{0}\right)} \simeq \bigoplus_{s_{1}+\cdots+s_{i} \leq l} V\left(s_{1} \omega_{1}+\cdots+s_{i} \omega_{i}\right)
$$


- $\mathrm{A}_{2 n-1}^{(2)}, \mathfrak{a}$ is of type $\mathrm{C}_{n}$

$$
\overline{V_{-\omega_{i}}\left(l \Lambda_{0}\right)} \simeq \bigoplus_{s_{p_{i}}+s_{p_{i}+2}+\cdots+s_{i}=l} V\left(s_{p_{i}} \omega_{p_{i}}+s_{p_{i}+2} \omega_{p_{i}+2}+\cdots+s_{i} \omega_{i}\right)
$$

- $\mathrm{D}_{n+1}^{(2)}, \mathfrak{a}$ is of type $\mathrm{B}_{n}$

$$
\begin{aligned}
& i=n: \overline{V_{-\omega_{i}}\left(l \Lambda_{0}\right)} \simeq V\left(l \omega_{n}\right) \\
& i \neq n: \overline{V_{-\omega_{i}}\left(l \Lambda_{0}\right)} \simeq \bigoplus_{s_{1}+\cdots+s_{i} \leq l} V\left(s_{1} \omega_{1}+\cdots+s_{i} \omega_{i}\right)
\end{aligned}
$$

- $\mathrm{E}_{6}^{(2)}, \mathfrak{a}$ is of type $\mathrm{F}_{4}$

$$
\begin{aligned}
& i=1: \overline{V_{-\omega_{i}}\left(l \Lambda_{0}\right)} \simeq \bigoplus_{0 \leq s \leq l} V\left(s \omega_{1}\right) \\
& i=4: \overline{V_{-\omega_{i}}\left(l \Lambda_{0}\right)} \simeq \bigoplus_{0 \leq s_{1}+s_{4} \leq l} V\left(s_{1} \omega_{1}+s_{4} \omega_{4}\right)
\end{aligned}
$$

- $\mathrm{D}_{4}^{(3)}, \mathfrak{a}$ is of type $\mathrm{G}_{2}$

$$
i=1: \overline{V_{-\omega_{i}}\left(l \Lambda_{0}\right)} \simeq \bigoplus_{0 \leq s \leq l} V\left(s \omega_{1}\right)
$$

For the other special vertices the decompositions can be computed by taking automorphisms.

Theorem 3 holds in the same way, for the basic module $W$ of the direct limit one choose $\bar{V}_{-\theta_{k}^{\vee}}\left(r \Lambda_{k}\right)$. Then the direct sum decomposition of $W$ contains obviously an one dimensional module, namely the one who corresponds in the Demazure module $V_{-\theta_{k}^{\vee}}\left(r \Lambda_{k}\right)$ to the weight $r \Lambda_{k}$. Again let $V_{\lambda, r}^{\infty}$ be the direct limit constructed above. Then it follows

Theorem 3. For any integral dominant weight $\Lambda$ of $\widehat{\mathfrak{g}}, \Lambda=r \Lambda_{k}+\lambda$, the a-modules $V_{\lambda, r}^{\infty}$ and $\bar{V}\left(r \Lambda_{k}\right)$ are isomorphic.

\section{REFERENCES}

[1] N. Bourbaki, Algèbre de Lie IV-VI, Hermann, Paris, 1968.

[2] V. Chari, On the fermionic formula and the Kirillov-Reshetikhin conjecture, Internat. Math. Res. Notices, 12 (2001), 629-654.

[3] M. Demazure, Une nouvelle formule de caractère, Bull. Sc. math., 98 (1974), 163-172. 
[4] G. Hatayama, A. Kuniba, M. Okado, T. Takagi and Z. Tsuboi, Paths, crystals and fermionic formula, MathPhys Odyssey 2001: Integrable Models and Beyond In Hormor of Barry M. McCoy, Birkhäuser (2002), pp. 205-272.

[5] G. Hatayama, A. N. Kirillov, A. Kuniba, M. Okado, T. Takagi and Y. Yamada, Character formulae of $\widehat{\mathfrak{s l}}_{n}$-modules and inhomogeneous paths, Nucl. Phys., B536 [PM] (1999), 575-616.

[6] J. Hong and S.-J. Kang, Introduction to Quantum Groups and Crystal Bases, Graduate Studies in Mathematics, Vol. 42, AMS, 2002.

[7] V. Kac, Infinite dimensional Lie algebras, third edition, Cambridge University Press, Cambridge, 1990.

[8] M. Kleber, Combinatorial structure of finite dimensional representations of Yangians, the simply-laced case, Int. Math. Res. Not., 7 (1997), no. 4, 187-2001.

[9] M. Kashiwara, On Level Zero Representations of Quantized Affine Algebras, Duke Math. J., 112 n.1 (2002), 117-175.

[10] M. Kashiwara, Level zero fundamental representations over quantized affine algebras and Demazure modules, Publ. Res. Inst. Math. Sci., 41 (2005), no. 1, 223-250.

[11] S. Kumar, Kac-Moody Groups, their flag varieties and representation theory, Progress in Mathematics, Birkhäuser Verlag, Boston, 2002.

[12] S. Kumar, Demazure Character Formula in arbitrary Kac-Moody setting, Invent. Math., 89 (1987), 395-423.

[13] A. Kuniba, K. Misra, M. Okado, T. Takagi, and J. Uchiyama, Crystals for Demazure Modules of Classical Affine Lie Algebras, J. Algebra, 208 (1998), no. 1, 185-215.

[14] A. Kuniba, K. Misra, M. Okado, T. Takagi, and J. Uchiyama, Demazure Modules and Perfect Crystals, J. Comm. Math. Phys., 192 (1998), 555-567.

[15] P. Littelmann, On spherical double cones, J. Algebra, 89 (1994), 142-157.

[16] P. Magyar, Littelmann Paths for the Basic Representation of an Affine Lie Algebra, math.RT/0308156.

[17] O. Mathieu, Formules de caractères pour les algèbres de Kac-Moody générales, Astérisque (1988), 159-160.

[18] S. Naito and D. Sagaki, Crystal of Lakshmibai-Seshadri Paths associated to an Integral Weight of Level Zero for an Affine Lie Algebra, math.QA/051001.

[19] S. Naito and D. Sagaki, Construction of perfect crystals conjecturally corresponding to Kirillov-Reshetikhin modules over twisted quantum affine algebras, math.QA/0503287.

[20] Y. Sanderson, Real characters for Demazure modules of rank two affine Lie algebras, J. Algebra, 184 (1996).

[21] S. Yamane, Perfect crystals of $U_{q}\left(\mathrm{G}_{2}^{(1)}\right)$, J. Algebra, 210 (1998), no. 2, 440-486.

\author{
G. Fourier \\ Mathematisches Institut der Universität Köln \\ Weyertal 86-90 \\ D-50931 Köln \\ Germany \\ gfourier@math.uni-koeln.de
}


P. Littelmann

Mathematisches Institut der Universität Köln

Weyertal 86-90

D-50931 Köln

Germany

littelmann@math.uni-koeln.de 